\title{
Cognitive tests used in chronic adult human randomised controlled trial micronutrient and phytochemical intervention studies
}

\author{
Anna L. Macready ${ }^{1}$, Laurie T. Butler ${ }^{1}$, , Orla B. Kennedy ${ }^{2}$, Judi A. Ellis ${ }^{1}$, Claire M. Williams ${ }^{1}$ \\ and Jeremy P. E. Spencer ${ }^{2}$ \\ ${ }^{1}$ School of Psychology and Clinical Language Sciences, University of Reading, Reading, UK \\ ${ }^{2}$ Hugh Sinclair Human Nutrition Unit, Department of Food Biosciences, University of Reading, Reading, UK
}

\begin{abstract}
In recent years there has been a rapid growth of interest in exploring the relationship between nutritional therapies and the maintenance of cognitive function in adulthood. Emerging evidence reveals an increasingly complex picture with respect to the benefits of various food constituents on learning, memory and psychomotor function in adults. However, to date, there has been little consensus in human studies on the range of cognitive domains to be tested or the particular tests to be employed. To illustrate the potential difficulties that this poses, we conducted a systematic review of existing human adult randomised controlled trial (RCT) studies that have investigated the effects of $24 \mathrm{~d}$ to 36 months of supplementation with flavonoids and micronutrients on cognitive performance. There were thirty-nine studies employing a total of 121 different cognitive tasks that met the criteria for inclusion. Results showed that less than half of these studies reported positive effects of treatment, with some important cognitive domains either under-represented or not explored at all. Although there was some evidence of sensitivity to nutritional supplementation in a number of domains (for example, executive function, spatial working memory), interpretation is currently difficult given the prevailing 'scattergun approach' for selecting cognitive tests. Specifically, the practice means that it is often difficult to distinguish between a boundary condition for a particular nutrient and a lack of task sensitivity. We argue that for significant future progress to be made, researchers need to pay much closer attention to existing human RCT and animal data, as well as to more basic issues surrounding task sensitivity, statistical power and type I error.
\end{abstract}

Cognitive tests: Micronutrients: Phytochemicals: Adult randomised controlled trials

\section{Introduction}

As the number of individuals over the age of 60 years is expected to double between 2000 and $2050^{(1)}$, the projected incidence of age-related neurodegenerative diseases and associated health care costs is also set to rise significantly. In a recent report commissioned by the Alzheimer's Society, the current annual cost of dementia in the UK alone was estimated to be about $£ 17.03$ billion $^{(2)}$, with the total worldwide cost estimated to be US\$315.4 billion annually $^{(3)}$. Moreover, given that individuals aged 65 years can now expect to live for at least another 20 years, there is an urgent need to identify means of mitigating age-related changes in healthy older adults. Diet is crucial in this respect as it is thought to reduce the impact of age-related cognitive decline, for instance, by combating oxidative stress, reducing LDL-cholesterol, and modulating neurological mechanisms such as cell-signalling pathways.

Over the last decade or so a significant, albeit mixed, body of evidence regarding the effects of diet on cognition has accumulated from human and animal work. For instance, in longitudinal and human observational studies, vitamin $\mathrm{E}$ intake has been associated with reduced agerelated cognitive decline through its antioxidant properties $^{(4)}$, and poorer memory performance has been linked to lower levels of serum vitamin E per unit of cholesterol ${ }^{(5)}$. Evidence from The Rotterdam Study has shown an association between higher plasma folate and better cognitive function, in particular for tests measuring psychomotor processing speed $^{(6)}$, episodic memory and

Abbreviations: ADAS-Cog, Alzheimer's Disease Assessment Scale Cognitive Subscale; category A, studies employing best designs and clear results; category B, studies with some weaknesses but no major problems; category C, studies showing significant methological and reporting problems; IQ, intelligence quotient; MMSE, Mini-Mental State Examination; RCT, randomised controlled trial.

* Corresponding author: Dr Laurie T. Butler, fax + 44118 9316715, email l.t.butler@ reading.ac.uk 
verbal ability ${ }^{(7)}$. In the PAQUID (Personnes Agées QUID, loosely translated to 'What about the elderly?') study, older adults with the highest dietary flavonoid intake showed significantly lower cognitive decline over 10 years than those with the lowest intake ${ }^{(8)}$. Studies using animal models have also demonstrated that certain groups of flavonoids may slow and even reverse the effects of ageing and dementia $^{(9-11)}$. For example, memory deficits may be prevented by the consumption of foods rich in anthocyanins, a flavonoid subgroup ${ }^{(12-16)}$.

While still an emerging area, an examination of the available human randomised controlled trial (RCT) literature reveals rather more variable evidence for the beneficial effects of diet on cognition. For example, in a systematic review of B vitamin and antioxidant supplement studies, Jia et al. ${ }^{(17)}$ found very little evidence for cognitive benefits from taking antioxidant supplements or B vitamins. A similar story is shown for flavonoid studies ${ }^{(18)}$, with reports of both significant ${ }^{(19-23)}$ and non-significant ${ }^{(24-29)}$ effects of supplementation.

Developing a better understanding of the conditions under which particular nutrients do or do not derive cognitive benefits represents a key challenge for research. However, one major problem facing researchers aiming to do this is that there is currently little consensus across studies in terms of either the cognitive domains to be explored or the specific tests to be used. Thus it is hard to determine whether a failure to reproduce a previously reported effect has established an important boundary condition for that nutrient (for example, supplementation with $\mathrm{X}$ not effective for population $\mathrm{Y}$ ) or, alternatively, is a reflection of the idiosyncrasies of the respective tasks employed across the two studies. For example, although it is often assumed that all tests of working memory performance reflect common mechanisms or processes, it is quite possible that different tests measure partially separate cognitive capacities ${ }^{(30)}$ and that performance dissociates across different tests. Indeed, Waters \& Caplan (2003) ${ }^{(31)}$ reported only moderate correlations between a series of seven different working memory measures. Thus simply assuming a one-to-one correspondence between two different cognitive measures purporting to measure the same domain (for example, working memory) is ill-advised. In the present paper we aim to establish the extent of this practice, as well as make recommendations for future studies.

\section{Cognitive domains and associated brain regions}

For the purposes of structuring the present review we now briefly outline the major taxonomies within human cognition. Importantly, attempts to characterise the effects of dietary nutrients on human cognition need to utilise a wide range of tasks to fully assess cognitive ability. In so doing, two points should be borne in mind. Firstly, although a particular task might be identified as having a primary neuropsychological focus such as 'executive function' or 'episodic memory', such measures are not 'task pure, ${ }^{(32)}$. For example, a range of processes may support a nominally 'executive' task such as memory, processing speed and motor function. Secondly, in terms of the underlying brain regions supporting cognitive performance, it is important to recognise that any task is likely to recruit multiple neural regions. For example, functional neuroimaging studies have revealed activations in the prefrontal cortex, medial and lateral parietal cortex, as well as hippocampal/medial temporal lobe activations during episodic memory retrie$\mathrm{val}^{(33)}$. A thorough understanding of the brain regions underpinning performance on particular cognitive tests is important, especially when attempting to relate findings from human studies to animal work. We return to this point in the Discussion.

\section{Executive function}

Executive function is a complex term used to describe a number of distinct, specifiable 'control' functions that are distinguishable from processing speed, memory, and motor functions. Examples of executive functions include 'switching' or 'shifting' (for example, alternating between behaviours or information sources), 'inhibition' (the ability to suppress automatic and habitual responses or behaviours), 'updating' (the ability to discard and replace information ${ }^{(34,35)}$ ), 'sustained attention' (requiring sustained concentration and monitoring skills ${ }^{(35,36)}$ ), 'strategic memory search' (conscious, controlled retrieval of structured information ${ }^{(32,35-37)}$ ), and 'planning' (the ability to deal with novel information, generate goals and make decisions on a suitable course of action ${ }^{(32,35)}$ ). Neuroimaging studies suggest that the prefrontal cortex and striatum interact to perform specific executive functions ${ }^{(38)}$, and that distinct brain regions are recruited for different executive functions. For instance, the left inferior frontal gyrus in the prefrontal cortex is recruited in verbal fluency tasks ${ }^{(39)}$, whereas the right inferior frontal gyrus shows greater activation in tasks measuring both shifting and inhibition $^{(38)}$.

\section{Working memory}

All of the above executive functions are dependent on 'working memory', a psychological construct used to describe a hypothetical system for the temporary maintenance and manipulation of speech-based and/or visuospatial information, requiring the control of attentional resources $^{(32)}$. Functional neuroimaging work shows that working memory is not a unitary or dedicated system, and is not localised to a single brain region ${ }^{(40)}$. D'Esposito described working memory as 'an emergent property of functional interactions between the PFC [prefrontal cortex] and the rest of the brain' (p. 769) ${ }^{(40)}$, and evidence suggests that the network of brain regions recruited for the active maintenance of task-relevant information will depend on the type of information being maintained ${ }^{(40,41)}$.

\section{Memory}

A number of key distinctions can be drawn between different types of memory. Specifically, researchers frequently distinguish between short-term memory (retrieval occurs within $30 \mathrm{~s}$ of stimulus presentation) v. long-term memory (retrieval occurs after 30s); explicit memory 
(consciously and intentionally retrieved) $v$. implicit memory (unconsciously retrieved); episodic (memory for events) $v$. semantic (memory for meaning); retrospective (memory for past events) $v$. prospective memory (remembering to perform actions in the future); memory for skills (procedural memory) $v$. memory for facts (declarative memory); and verbal memory $v$. visual or visuospatial memory.

As might be expected, a wide range of brain regions are thought to be involved in supporting these various forms of memory. For instance, activation of left-lateralised posterior temporal regions, the supramarginal gyrus, dorsolateral premotor cortex and Broca's area have been associated with short-term memory ${ }^{(42)}$, in contrast to activation of bilateral ventrolateral prefrontal regions and dorsolateral prefrontal regions during encoding and recognition in long-term episodic memory ${ }^{(43)}$. In terms of the neural substrate of explicit and implicit memory tasks ${ }^{(44)}$, explicit memory has been linked to left frontal, and bilateral hippocampal, parahippocampal and parietal activation ${ }^{(45)}$, whereas implicit memory is primarily associated with reduced left fusiform gyrus and bilateral frontal and occipital activity $^{(46,47)}$. The hippocampus, parahippocampus and parietal areas are typically implicated in spatial memory tasks $^{(48)}$, whereas the anterior prefrontal cortex has been shown to be actively involved in prospective memory $\operatorname{tasks}^{(49)}$.

\section{Motor function, perception and intelligence quotient}

Motor function may be measured with or without a cognitive component, and encompasses a range of measures from psychomotor processing speed to planning of movement. Voluntary movement is controlled by the basal ganglia system, which includes the striatum and substantia nigra, by enabling required motor mechanisms and inhibiting competing mechanisms ${ }^{(50)}$. Various brain regions are thought to be involved during motor skill acquisition: prefrontal regions are recruited initially, with a subsequent shift to posterior regions, for example, premotor, posterior parietal and cerebellar cortex structures, as the task becomes more automatic ${ }^{(51)}$.

Visual perception relies on visual acuity, field of view and contrast sensitivity, abilities that are reduced with age ${ }^{(52,53)}$ and which underpin any cognitive function with a visual component. Visual perception is associated with a wide range of brain regions in neuroimaging studies, namely the striate cortex and other occipital areas, parietal, temporal and prefrontal regions ${ }^{(54)}$.

Intelligence tasks may be sub-divided into crystallised intelligence (measuring acquired knowledge) and fluid intelligence (measuring non-verbal ability, problem-solving and pattern recognition independently of acquired knowledge $)^{(55)}$. General intelligence, or Spearman's $g$, is associated most closely with fluid intelligence and activation of the lateral frontal ${ }^{(56)}$ or prefrontal cortex and parietal areas ${ }^{(57)}$.

\section{Research aims and questions}

The primary aim of the present paper is to review the cognitive methods used in existing RCT studies that have explored the effects of nutrition on human cognition, with a view to identifying domains (for example, executive function, episodic memory) and individual tasks within those domains (for example, category fluency task, common objects recall task) that have shown greatest sensitivity to chronic supplementation (for example, supplementation of a nutrient over a number of days, weeks or months, as opposed to an acute intake of a nutrient on a single experimental day). A related aim is to catalogue the cognitive tasks used in existing chronic RCT studies within a single framework enabling researchers to better choose suitable tasks, as well as identify potential gaps in terms of the domains measured.

It should be noted here that significant outcomes for cognitive testing in dietary intervention studies rely on two things: (1) the potential for cognitive change as a result of direct dietary intervention with respect to dose and duration in the cognitive domain or cognitive aspect being measured and (2) cognitive methodologies sensitive enough to measure such cognitive change. The most important consideration in setting up a suitable framework for measuring human cognitive function in nutritional research is to determine methods that are sensitive to dietary changes and repeatable over time, are simple to interpret, and specific to cognitive domains. In this respect, brief measures such as the Mini-Mental State Examination (MMSE) ${ }^{(58)}$ and the Alzheimer's Disease Assessment Scale Cognitive Subscale (ADAS-Cog) ${ }^{(59)}$ are suitable for cognitive screening of dementia and mild cognitive impairment, a term generally used to describe the level of cognitive impairment found in the intermediate stage between normal ageing and fully developed dementia ${ }^{(60)}$. Both the MMSE and the ADAS-Cog consist of items covering a broad range of cognitive functions: orientation, attention and calculation, memory, language, and motor skills, but they cannot truly be said to measure 'global cognitive function', as the individual test items do not measure the full range of cognitive functions. The term 'general cognitive function' is therefore preferred here.

In terms of examining changes in cognitive performance over time, the MMSE and ADAS-Cog may be useful for the measurement of widespread, gross cognitive changes in longitudinal studies ${ }^{(8)}$; in Alzheimer's disease research, where the fastest rate of deterioration over time is likely to be seen, the MMSE has shown an overall progression rate of 0.24 points per month, although this was moderated by education duration, sex, disease incidence and drug therapy ${ }^{(61)}$. However, such measures are unlikely to be sensitive to smaller changes over shorter time periods in healthier individuals at pre-dementia stages, and will indeed show ceiling effects in young and/or cognitively healthy adult populations.

Overall we address four main questions:

(1) What proportion of chronic dietary interventions has reported significant benefits to cognition and in which domains?

(2) How much consistency is there across studies in terms of cognitive domains measured and tasks employed?

(3) Are there any cognitive domains that are underrepresented in existing intervention studies? 
(4) What are the implications for future chronic dietary intervention studies?

\section{Methods}

A search of five databases (PUBMED, Web of Knowledge, PsychINFO, CINAHL and the Cochrane Central Register of Controlled Trials) was carried out for micronutrient or phytochemical adult human randomised controlled intervention trials exploring cognitive function as the primary or secondary outcome. The following search terms were used:

Cognitive tasks: cogniti*, executive function, switching, shifting, updating, inhibition, vigilance, attention, memory, episodic, semantic, implicit, explicit, spatial, visuospatial, prospective, declarative, procedural, processing speed, psychomotor, reaction time, accuracy.

(1) Nutrient: vitamin*, thiamin*, riboflavin, niacin, nicotinamide, pantothenic, pyridox*, biotin, cobalamin*, folic acid, folate, ascorbic, tocopherol, iron, copper, zinc, magnesium, manganese, selenium, flavan*, flavon*, isoflavone, caroten*.

(2) Population sample: human adult [not] adolescent, child, infant, maternal, rat, mouse, mice, rodent, dog, monkey.

(3) Experimental design: randomi*, controlled trial, RCT.

(4) Type of article: journal article, peer-reviewed.

All studies identified through the literature search were evaluated according to the eligibility criteria by one reviewer and independently verified by a second reviewer. The search was limited to the previous 10 years (for example, 1 January 1999 to 31 August 2009).

Chronic studies that specifically focused on the cognitive effects of the target nutrients, both single and combined, were included. Studies exploring phytochemicals administered in extract rather than whole-food form (for example, Ginkgo biloba) were included. Studies on populations suffering from age-related cognitive impairment or dementia were included, except in the case of traumatic head injury or specific neurological disorders, whose findings may not be generalisable to a normal human adult population.

Acute interventions, non-randomised studies, RCT with sample sizes of less than twenty participants (or less than ten for cross-over designs), and studies that did not include a proper baseline, or control and/or placebo group were excluded. Studies using whole foods or treatments combined only with macronutrients or drug therapy were also excluded, as were drug therapy studies using micronutrients as a placebo control. As the present review is focusing on micronutrient or phytochemical strategies for the attenuation and prevention of cognitive decline throughout the adult lifespan, cognitive development studies (for example, maternal, infant and child) were excluded. As we were primarily interested in comparing uniquely human, language-based cognitive paradigms across studies, animal studies were excluded. Also excluded were studies of specific hospital-based patient groups (for example, CHD, stroke or diabetes), as were studies comparing pre- and

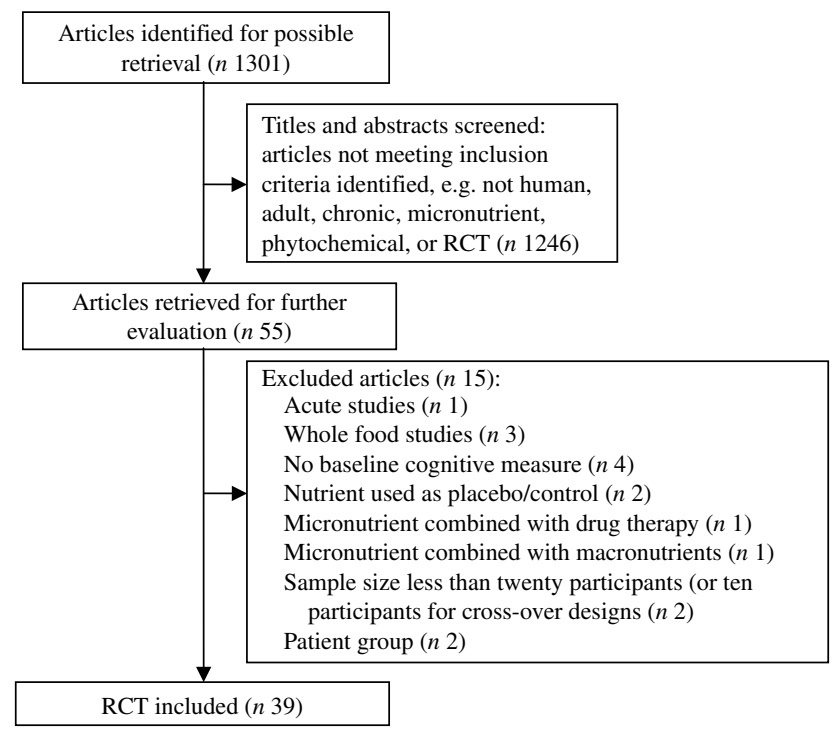

Fig. 1. Randomised controlled trial (RCT) literature search and screening process.

post-operative cognitive performance. The literature search and screening process is shown in Fig. 1.

Outcomes were changes in cognitive performance. The first author categorised the tasks and the second author resolved any inconsistencies encountered during classification. Tasks were initially categorised by their primary neuropsychological focus, which was determined by the descriptions provided in the selected papers. Where disagreement occurred, the authors used the task descriptions cited in the majority of the included studies and checked these, where possible, against Lezak et al. ${ }^{(62)}$, a comprehensive sourcebook of neuropsychological assessment.

All included studies were rated using a three-category quality assessment grading system (A, B, C), based on a method successfully trialled elsewhere ${ }^{(63,64)}$, to identify any methodological shortcomings which might affect interpretation of the results. Briefly, category A studies employ the best designs, such that they are sufficiently powered with less than $20 \%$ drop-out, methods (double-blind, intervention, comparator, outcome measures and statistical tests) are appropriate, results are clearly reported and assessed as valid. Category B studies may contain some weaknesses, but show no major problems and are still considered valid. Category C studies show significant methodological difficulties which may invalidate results, with flawed designs and analysis, missing information, greater than $20 \%$ drop-out, randomisation issues (for example, unequal between-group baseline scores), reporting discrepancies and low power. Grading was carried out and agreed by the first and second authors.

Studies were examined for cognitive outcome status in order to see if the RCT was designed primarily to test cognitive function, or if cognition was only a secondary outcome measure, as this again may affect the validity of the results. Cognitive outcome status was then designated as 'primary' or 'secondary' for all RCT. 


\section{Results}

\section{Assessment of cognitive performance in existing studies}

Thirty-nine studies met the inclusion criteria (see Table 1). Five used multivitamins ${ }^{(65-69)}$, and two of these also included minerals ${ }^{(67,68)}$. Ten studies examined vitamin B treatments ${ }^{(70-79)}$ and three looked at specific minerals: $\mathrm{Zn}^{(80)}, \mathrm{Fe}^{(81)}$ and $\mathrm{Cu}^{(82)}$. One trialled $\beta$-carotene with vitamins $\mathrm{C}$ and $\mathrm{E}^{(83)}$. Of the studies targeting individual micronutrients or phytochemicals, twenty looked at flavonoids ${ }^{(19-29,84-92)}$. Twelve of these were isoflavone $\mathrm{RCT}^{(19-22,24,25,27,28,84-86,89)}$, six used G. biloba extracts containing 24 or $25 \%$ flavonoids and $6 \%$ terpenes ${ }^{(23,26,29,88,91,92)}$, one used pine bark ${ }^{(90)}$ and one used cocoa flavanols ${ }^{(87)}$. No other intervention met the study criteria within the time frame of the review.

Fifteen studies $(38 \%)$ were graded as category $\mathrm{C}$ and judged to contain significant bias that may invalidate the results, mostly as a result of lack of any quantitative cognitive screening, missing information and reporting errors. Eighteen studies $(46 \%)$ were classed as category B and judged to be susceptible to bias, but not sufficiently so to invalidate the results. Only six studies $(15 \%)$ met the more rigorous criteria for category A, as described earlier (see Table 1). In the assessment of cognitive outcome status, $69 \%$ of the RCT were found to be specifically designed to measure cognitive function as the primary outcome (see Table 1).

Only seventeen studies (44\%) reported benefits of treatment on cognitive function in the expected direction $^{(19-23,65,68,75,77,79,80,84,86,89-92)}$, of which two were graded as category $\mathrm{A}^{(77,80)}$, seven were category $\mathrm{B}^{(21-23,65,79,86,90)}$ and the rest were category C. Twelve of the seventeen RCT were flavonoid ${ }^{(19-23,84,86,88-92)}$ including seven isoflavone studies ${ }^{(19-22,84,86,89)}$ and four G. biloba interventions ${ }^{(23,88,91,92)}$.

In evaluating these effects of treatment, there was found to be considerable variability in the statistical rigour employed in individual studies. Gleason et al. ${ }^{(20)}$ reported both positive and negative effects with a small sample size of thirty, but did not appear to have accounted for the possibility of type I error. Mix \& Crews ${ }^{(8)}$ reported a small significant effect of treatment on a single outcome measure using a one-tailed $t$ test, a result unlikely to survive cut-off if an arguably more appropriate two-tailed convention had been employed. Additionally, both Casini et al. ${ }^{\left({ }^{(4)}\right.}$ and Santos et al. ${ }^{(91)}$ reported multiple $t$ tests without any apparent correction for type I error. Howes et al. ${ }^{(85)}$ also carried out a large number of tests on a small sample ( $n$ 30) initially reporting a series of significant effects. However, as an illustration of good practice, these disappeared after the authors statistically accounted for type I error. Finally, Stough et al. ${ }^{(92)}$ provided no descriptive statistics at all, making it impossible to evaluate the quality or rigour of their experimental design and analysis.

Of the micronutrient studies, three vitamin B studies reported some positive effects of treatment ${ }^{(75,77,79)}$, although Bryan et al. ${ }^{(79)}$ also reported negative effects. Two multivitamin interventions reported benefits ${ }^{(65,68)}$, and Maylor et al. ${ }^{(80)}$ found both positive and negative effects of $\mathrm{Zn}$ treatment on cognitive function.
Interestingly, four studies (10\%) showed only null and negative effects of treatment on cognitive function: three vitamin B studies $^{(72,73,78)}$ and one flavonoid intervention ${ }^{(27)}$. The vitamin B studies were carried out on older populations and appear to have used $t$ tests on multiple tasks with no correction for type I error.

Of the thirty-nine studies included in the present review, the size of study populations ranged from sixteen in a functional magnetic resonance imaging cross-over study ${ }^{(87)}$ to $818^{(77)}$. Seventeen studies had fewer than 100 participants, and five studies had forty participants or less ${ }^{(20-22,26,85)}$. Power calculations were carried out in only four RCT, all researching vitamin $\mathrm{B}$, with populations of 179 or more m $^{(71,72,75,76)}$, but these were based largely on expected changes in physiological rather than cognitive markers. In other RCT, group size does not appear to have been driven by effect sizes found in previous studies and varies considerably across nutrient studies, for example Pathansali et al. ${ }^{(73)}$ with groups of twelve participants, and Durga et al. ${ }^{(77)}$ with groups of over 400.

Participant ages were highly variable, ranging from 18 years to over 80 years of age, with twenty-nine studies (74\%) carried out on participants over the age of 50 years, including nine studies specifically carried out on adults of 65 years or more. Three further RCT included young and older adult populations ${ }^{(23,25,79)}$, two more studies focused on the 40-65 years age range $\mathrm{e}^{(27,84)}$ and five others were conducted on 18- to 40 -year-olds ${ }^{(21,26,81,87,92)}$.

\section{Single v. multiple cognitive domains}

Among the thirty-nine RCT included in the present review, a variety of approaches was used to measure cognitive performance, mostly targeting multiple cognitive domains, with the exception of a flavonoid brain imaging study which used a single executive function 'switching' (or 'shifting') task $^{(87)}$. Of the RCT testing multiple cognitive domains, the majority examined a range of specific memory processes and executive functions. The remaining RCT targeted general cognition rather than any specific domain (see Table 1).

\section{Rationale for choice of cognitive tests}

Twenty-one RCT based their choice of cognitive test(s) on findings from previous studies. Eleven cited sensitivity to the class of nutrient under investigation, such as B vitamins, flavonoids, other type of dietary manipulation, hormone replacement therapy or oestrogen $^{(19,21,22,27,28,65,68,71,73,79,83)}$. Seven studies instead used tasks sensitive to ageing ${ }^{(29,77,85)}$, brain disorders and pharmacological interventions ${ }^{(23,76,80)}$, or changes in functional magnetic resonance imaging blood oxygenation level-dependent (BOLD) signal ${ }^{(87)}$. Three RCT selected tasks from established computerised psychometric test series: Ryan et al. ${ }^{(90)}$ used the Cognitive Drug Research ${ }^{\circledR}$ battery ${ }^{(93)}$; Murray-Kolb \& Beard ${ }^{(81)}$ the Cognitive Abilities Test battery ${ }^{(94)}$; and Mix \& Crews ${ }^{(88)}$ selected the TrailMaking Test on the basis that it appeared to be 'one of the best measures of general cognitive functioning' (p. 223) according to Reitan ${ }^{(95)}$. These tests were developed for use 


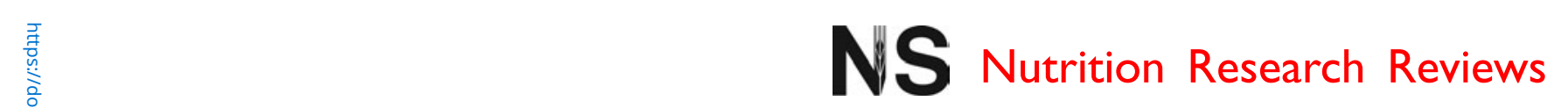

Table 1. Continued

\begin{tabular}{|c|c|c|c|c|c|c|c|c|c|}
\hline Nutrient & Authors & Subjects $(n)$ & Age (years) & $\begin{array}{l}\text { Life stage } \\
\text { and/or sex }\end{array}$ & Setting & $\begin{array}{l}\text { Mental, nutrient } \\
\text { or health status }\end{array}$ & $\begin{array}{l}\text { Design, outcome } \\
\text { statusł and category§ }\end{array}$ & Intervention, dose and duration & Key findings \\
\hline Multivitamin & $\begin{array}{l}\text { Wouters- } \\
\text { Wesseling } \\
\text { etal. (2005) }\end{array}$ & $\begin{array}{l}\text { ITT: } 101 \\
\text { (D/O: 34/101) }\end{array}$ & $65+$ & Older adult & $\begin{array}{l}\text { The Nether- } \\
\text { lands }\end{array}$ & $\begin{array}{c}\mathrm{T}: 21 \% \mathrm{MMSE} \leq 23 \\
\mathrm{C}: 24 \% \mathrm{MMSE}=23 \\
\mathrm{BMI}<25 \mathrm{~kg} / \mathrm{m}^{2} \\
\text { 10-36\% below RDA } \\
\quad \text { for vitamins } \mathrm{B}_{1}, \mathrm{~B}_{2}, \\
\mathrm{~B}_{6}, \mathrm{~B}_{12} \text { and folate }\end{array}$ & $\begin{array}{l}\text { Design: double-blind random- } \\
\quad \text { ised placebo-controlled } \\
\text { Outcome: primary } \\
\text { Category: C }\end{array}$ & $\begin{array}{l}\text { T: } 125 \mathrm{ml} \text { enriched drink containing } \\
30-50 \% \text { of the US RDA of } \\
\text { vitamins and minerals with } \\
\text { enhanced amounts of antioxi- } \\
\text { dants and } 250 \mathrm{kcal} \text { (1046 kJ) } \\
\text { energy (once daily) } \\
\text { C: placebo (unspecified - twice } \\
\text { daily) } \\
\text { Duration: } 6 \text { months }\end{array}$ & $\begin{array}{l}\text { General: } \\
\text { MMSE (screening) } \\
\text { Memory: } \\
\text { Fifteen-Word Learning Test }\left({ }^{\star} P<0.05 \text { ) }\right. \\
\text { Recognition Memory Test for Words (NS) } \\
\text { Executive function/working memory: } \\
\text { Category Fluency Test (Animals/ } \\
\quad \text { Professions) }\left({ }^{*} P<0.05 \text { ) }\right.\end{array}$ \\
\hline Vitamin B & $\begin{array}{l}\text { Aisen et al. } \\
\qquad(2008)^{(76)}\end{array}$ & $\begin{array}{l}306 \\
\text { ITT: } 409\end{array}$ & $50+$ & $\begin{array}{l}\text { Alzheimer's dis- } \\
\text { ease, } \\
\text { older adult }\end{array}$ & USA & $\begin{array}{l}\text { MMSE } 14-26 \\
\text { Mild to moderate } \\
\text { Alzheimer's } \\
\text { disease }\end{array}$ & $\begin{array}{l}\text { Design: double-blind random- } \\
\quad \text { ised placebo-controlled } \\
\text { Outcome: secondary } \\
\text { Category: B }\end{array}$ & $\begin{array}{l}\text { T: } 5 \mathrm{mg} \text { folate/d, } 25 \mathrm{mg} \text { vitamin } \mathrm{B}_{6} / \mathrm{d} \\
\quad \text { and } 1 \mathrm{mg} \text { vitamin } \mathrm{B}_{12} / \mathrm{d} \\
\text { C: placebo (unspecified) } \\
\text { Duration: } 18 \text { months }\end{array}$ & $\begin{array}{l}\text { General: } \\
\text { ADAS-cog (NS) } \\
\text { MMSE (NS) (screening and performance } \\
\text { measure) } \\
\text { Clinical Dementia Rating Sum of Boxes (NS) }\end{array}$ \\
\hline Vitamin B & $\begin{array}{l}\text { Bryan et al. } \\
\quad(2002)^{(79)}\end{array}$ & $\begin{array}{l}211 \\
\text { ITT: } 221\end{array}$ & $\begin{array}{l}20-30 \\
45-55 \\
65-92\end{array}$ & $\begin{array}{l}\text { Adult female, } \\
\text { older adult } \\
\text { female }\end{array}$ & Australia & $\mathrm{n} / \mathrm{a}$ & $\begin{array}{l}\text { Design: double-blind random- } \\
\text { ised placebo-controlled } \\
\text { Outcome: primary } \\
\text { Category: B }\end{array}$ & $\begin{array}{l}\text { T1: } 750 \mu \mathrm{g} \text { folate/d } \\
\text { T2: } 15 \mu \mathrm{g} \text { vitamin } \mathrm{B}_{12} / \mathrm{d} \\
\text { T3: } 75 \mathrm{mg} \text { vitamin } \mathrm{B}_{6} / \mathrm{d} \\
\text { C: placebo (unspecified) } \\
\text { Duration: } 5 \text { weeks }\end{array}$ & $\begin{array}{l}\text { Memory: } \\
\text { Activity Recall (NS) } \\
\text { Digit-Symbol-Coding Task, Symbol Recall } \\
\text { (WAIS) (NS) } \\
\text { Rey Auditory-Verbal Learning Test } \\
\left(^{*} P<0.05\right) \\
\text { Self-Ordered Pointing Task (NS) } \\
\text { Vocabulary (WAIS) (NS) } \\
\text { Executive function/working memory: } \\
\text { Digit Span Backwards (WAIS) (NS) } \\
\text { Letter-Number Sequencing (NS) } \\
\text { Stroop Colour-Word Test (NS) } \\
\text { Symbol Search (WAIS) (NS) } \\
\text { Spot The Word (NS) } \\
\text { Trail-Making Test (HRNTB) (NS) } \\
\text { Uses for Common Objects (NS) } \\
\text { Verbal Fluency (Initial Letter) († } P<0.05 \text { ) } \\
\text { Verbal Fluency (Excluded Letter) (NS) } \\
\text { Psychomotor function/processing speed: } \\
\text { Boxes Test (NS) } \\
\text { Digit Symbol-Coding (WAIS) (NS) }\end{array}$ \\
\hline Vitamin B & $\begin{array}{l}\text { Durga et al. } \\
\quad(2007)^{(77)}\end{array}$ & ITT: 818 & $50-70$ & Older adult & $\begin{array}{l}\text { The Nether- } \\
\text { lands }\end{array}$ & $\begin{array}{l}\text { MMSE } \geq 24 \\
\text { Homocysteine } \\
13-26 \mu \mathrm{mol} / \mathrm{l} \\
\text { Vitamin } \\
\mathrm{B}_{12}<200 \mathrm{pmol} / \mathrm{l}\end{array}$ & $\begin{array}{l}\text { Design: double-blind random- } \\
\text { ised placebo-controlled } \\
\text { Outcome: primary } \\
\text { Category: A }\end{array}$ & $\begin{array}{l}\mathrm{T}: 800 \mu \mathrm{g} \text { folic acid/d } \\
\text { C: placebo (unspecified) } \\
\text { Duration: } 36 \text { months }\end{array}$ & $\begin{array}{l}\text { General: } \\
\text { MMSE (screening) } \\
\text { Global Cognitive Score }\left({ }^{\star} P<0.05\right) \\
\text { Memory: } \\
\text { Thirty-Word Learning Test }\left({ }^{\star} P<0.05\right) \\
\text { Executive function/working memory: } \\
\text { Concept Shifting Test (NS) } \\
\text { Stroop Colour-Word Test (NS) } \\
\text { Verbal Fluency Test (NS) } \\
\text { Psychomotor function/processing speed: } \\
\text { Letter Digit Substitution Test }\left({ }^{\star} P<0.05 \text { ) }\right. \\
\text { Stroop Colour-Word Test (word } \\
\text { reading) (NS) } \\
\text { Stroop Colour-Word Test (naming ink } \\
\text { colour) (NS) }\end{array}$ \\
\hline
\end{tabular}




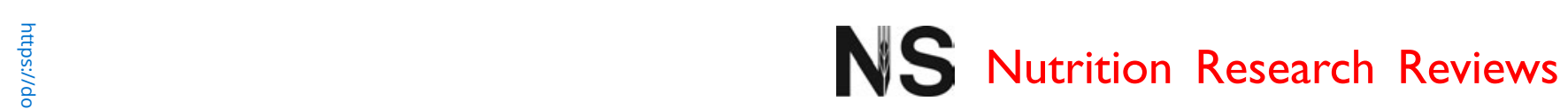

\begin{tabular}{|c|c|c|c|c|c|c|c|c|c|}
\hline Vitamin B & $\begin{array}{l}\text { Eussen et al. } \\
\qquad(2006)^{(71)}\end{array}$ & 195 & $70+$ & Older adult & $\begin{array}{l}\text { The Nether- } \\
\text { lands }\end{array}$ & $\begin{array}{l}\text { MMSE }>19 \\
\text { Randomisation strati- } \\
\text { fied according to } \\
\text { sex, methylmalonic } \\
\text { acid concentration } \\
\text { at screening and } \\
\text { MMSE }<\text { and }>24 \\
\text { Mild } B_{12} \text { deficiency }\end{array}$ & $\begin{array}{l}\text { Design: double-blind random- } \\
\text { ised placebo-controlled } \\
\text { Outcome: primary } \\
\text { Category: A }\end{array}$ & $\begin{array}{l}\text { T1: } 1000 \mathrm{mg} \text { vitamin } B_{12} / \mathrm{d} \\
\text { T2: } 1000 \mathrm{mg} \text { vitamin } B_{12} / \mathrm{d} \text { and } \\
\quad 400 \mathrm{mg} \text { folic acid/d } \\
\text { C: placebo (unspecified) } \\
\text { Duration: } 24 \text { months }\end{array}$ & $\begin{array}{l}\text { IQ: } \\
\text { Raven's Progressive Matrices } \\
\text { General: } \\
\text { MMSE (NS) (screening and performance } \\
\text { measure) } \\
\text { Memory: } \\
\text { 15-Word Learning Test (NS) } \\
\text { Digit Span Forward (WAIS) (NS) } \\
\text { Similarities Test (WAIS) (NS) } \\
\text { Word Recognition (NS) } \\
\text { Executive function/working memory: } \\
\text { Digit Span Backward (WAIS) (NS) } \\
\text { Rey-Osterreith Complex Figure Test (NS) } \\
\text { Stroop Colour-Word Test (NS) } \\
\text { Trail-Making Test (HRNTB) (NS) } \\
\text { Verbal Fluency (Initial Letter) (NS) } \\
\text { Verbal Fluency (Animals) (NS) } \\
\text { Psychomotor function/processing speed: } \\
\text { Finger-Tapping Task (NS) } \\
\text { Psychomotor Planning (NS) }\end{array}$ \\
\hline Vitamin B & $\begin{array}{l}\text { Hvas et al. } \\
\qquad(2004)^{(70)}\end{array}$ & 140 & Mean 74.5 & Older adult & Denmark & $\begin{array}{l}29 \% \text { MMSE }<25 \\
36 \% \text { MMSE }<2656 \% \\
\text { CAMCOG }<90 \\
\text { (mean } 89 \text { ) } \\
27 \% \text { low scores in both }\end{array}$ & $\begin{array}{l}\text { Design: double-blind random- } \\
\text { ised placebo-controlled } \\
\text { Outcome: primary } \\
\text { Category: A }\end{array}$ & $\begin{array}{l}\mathrm{T}: 1 \mathrm{mg} \text { vitamin } \mathrm{B}_{12} / \text { week } \\
\text { (BetolvexR) } \\
\text { C: placebo ( } 1 \mathrm{ml} \text { isotonic sodium } \\
\text { chloride/week) } \\
\text { Duration: } 3 \text { months }\end{array}$ & $\begin{array}{l}\text { General: } \\
\text { CAMCOG (NS) } \\
\text { MMSE (NS) (screening and performance } \\
\text { measure) } \\
\text { Memory: } \\
\text { 12-Words Learning Test (NS) }\end{array}$ \\
\hline Vitamin B & $\begin{array}{l}\text { Lewerin et al. } \\
\quad(2005)^{(78)}\end{array}$ & 209 & Mean 76.4 & Older adult & Sweden & $n / a$ & $\begin{array}{l}\text { Design: double-blind random- } \\
\text { ised placebo-controlled } \\
\text { Outcome: secondary } \\
\text { Category: B }\end{array}$ & $\begin{array}{l}\mathrm{T}: 0.5 \mathrm{mg} \text { vitamin } \mathrm{B}_{1} / \mathrm{d}, 0.8 \mathrm{mg} \text { folic } \\
\quad \text { acid/d and } 3 \mathrm{mg} \text { vitamin } \mathrm{B}_{6} / \mathrm{d} \\
\text { C: placebo (unspecified) } \\
\text { Duration: } 4 \text { months }\end{array}$ & $\begin{array}{l}\text { Memory: } \\
\text { Digit Span Forwards (WAIS) (NS) } \\
\text { Synonyms ( } P<0.05 \text { ) } \\
\text { Thurstone's Picture Memory Test (NS) } \\
\text { Visual reproduction (WMS) (NS) } \\
\text { Executive function/working memory: } \\
\text { Block design (WAIS) (NS) } \\
\text { Digit Span Backwards (WAIS) (NS) } \\
\text { Figure classification (NS) } \\
\text { Psychomotor function/processing speed: } \\
\text { Digit Symbol (WAIS) (NS) } \\
\text { Identical forms († } P<0.05 \text { ) } \\
\text { Postural-Locomotor-Manual Test (NS) }\end{array}$ \\
\hline Vitamin B & $\begin{array}{l}\text { McMahon et al. } \\
\quad(2006)^{(72)}\end{array}$ & 253 & $65+$ & Older adult & New Zealand & $\begin{array}{l}\text { Plasma homocysteine } \\
\text { concentrations } \geq \\
13 \mu \mathrm{mol} / \mathrm{l}\end{array}$ & $\begin{array}{l}\text { Design: double-blind random- } \\
\text { ised placebo-controlled } \\
\text { Outcome: secondary } \\
\text { Category: B }\end{array}$ & $\begin{array}{l}\text { T: } 1000 \mu \mathrm{g} \text { folate/d, } 500 \mu \mathrm{g} \text { vitamin } \\
\mathrm{B}_{1} / \mathrm{d} \text { and } 10 \mathrm{mg} \text { vitamin } \mathrm{B}_{6} / \mathrm{d} \\
\text { C: placebo (magnesium stearate }+ \\
\text { microcrystalline cellulose) } \\
\text { Duration: } 24 \text { months }\end{array}$ & $\begin{array}{l}\text { IQ: } \\
\text { National Adult Reading Test } \\
\text { Raven's Progressive Matrices } \\
\text { General: } \\
\text { MMSE (NS) } \\
\text { Memory: } \\
\text { Paragraph Recall Test }(\dagger P<0.05) \\
\text { Rey Auditory-Verbal Learning Test (NS) } \\
\text { Executive function/working memory: } \\
\text { Category Word Fluency Test (NS) } \\
\text { Controlled Oral Word Association Test (NS) } \\
\text { Trail-Making Test (HRNTB) }(\dagger P<0.05)\end{array}$ \\
\hline
\end{tabular}




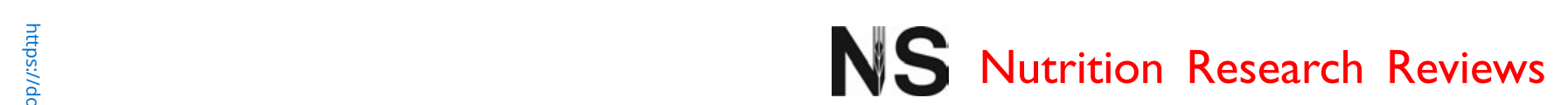

Table 1. Continued

\begin{tabular}{|c|c|c|c|c|c|c|c|c|}
\hline Nutrient & Authors & Subjects $(n)$ & Age (years) & $\begin{array}{l}\text { Life stage } \\
\text { and/or sex }\end{array}$ & Setting & $\begin{array}{l}\text { Mental, nutrient } \\
\text { or health status }\end{array}$ & $\begin{array}{l}\text { Design, outcome } \\
\text { statusł and category§ }\end{array}$ & Intervention, dose and duration \\
\hline Vitamin B & $\begin{array}{c}\text { Pathansali et al. } \\
(2006)^{(73)}\end{array}$ & 24 & $65+$ & Older adult & UK & MMSE $\geq 27$ & $\begin{array}{l}\text { Design: double-blind random- } \\
\text { ised placebo-controlled } \\
\text { Outcome: primary } \\
\text { Category: B }\end{array}$ & $\begin{array}{l}\text { T: } 5 \mathrm{mg} \text { folic acid/d } \\
\text { C: placebo (unspecified) } \\
\text { Duration: } 4 \text { weeks }\end{array}$ \\
\hline Vitamin B & $\begin{array}{l}\text { Seal et al. } \\
(2002)^{(74)}\end{array}$ & $\begin{array}{l}26 \\
\text { (D/O: } 5 / 31)\end{array}$ & Mean $84 \cdot 1$ & Older adult & Australia & $\begin{array}{l}\text { MMSE: } 6-28 \text { (about } \\
33 \% \text { had dementia) } \\
\text { Low vitamin } \mathrm{B}_{12} \\
\text { About } 50 \% \text { cerebro- } \\
\text { vascular disease or } \\
\text { CVD } \\
\text { About } 11 \% \text { type } 2 \\
\text { diabetes }\end{array}$ & $\begin{array}{l}\text { Design: double-blind random- } \\
\text { ised placebo-controlled } \\
\text { Outcome: secondary } \\
\text { Category: B }\end{array}$ & $\begin{array}{l}\text { T1: } 10 \mu \mathrm{g} \text { vitamin } \mathrm{B}_{12} / \mathrm{d} \\
\mathrm{T} 2: 50 \mu \mathrm{g} \text { vitamin } \mathrm{B}_{12} / \mathrm{d} \\
\mathrm{C}: \text { placebo (daily } 40 \mathrm{ml} \text { Australian } \\
\text { Pharmaceutical Formulary red } \\
\text { mixture }+2 \mathrm{ml} \text { hydrobenzoate } \\
\text { compound) } \\
\text { Duration: } 1 \text { month }\end{array}$ \\
\hline Vitamin B & $\begin{array}{l}\text { van Uffelen et al. } \\
\quad(2008)^{(75)}\end{array}$ & $\begin{array}{l}\text { ITT: } 179 \\
\text { mITT: 152 } \\
\text { (D/O: 13/152) }\end{array}$ & $70-80$ & $\mathrm{MCl}$, older adult & $\begin{array}{l}\text { The Nether- } \\
\text { lands }\end{array}$ & $\begin{array}{l}\text { MMSE } \geq 24 \\
\text { TICS } \geq 19 \\
\text { Word Learning Test } \\
\quad \text { Delayed } \\
\text { Recall } \leq 5 / 10\end{array}$ & $\begin{array}{l}\text { Design: double-blind random- } \\
\text { ised placebo-controlled } \\
\text { Outcome: secondary } \\
\text { Category: C }\end{array}$ & $\begin{array}{l}\text { T: } 5 \mathrm{mg} \text { folic acid/d, } 0.4 \mathrm{mg} \text { vitamin } \\
\mathrm{B}_{1 / 2} / \mathrm{d} \text { and } 50 \mathrm{mg} \text { vitamin } \mathrm{B}_{6} / \mathrm{d} \\
\text { C: placebo (unspecified) } \\
\text { Duration: } 12 \text { months }\end{array}$ \\
\hline $\begin{array}{l}\text { Vitamin C/ } \\
\text { vitamin E/ } \\
\text { carotenoid }\end{array}$ & $\begin{array}{l}\text { Smith et al. } \\
(1999)^{(83)}\end{array}$ & 205 & $60-80$ & Older adult & UK & $\begin{array}{l}\text { MMSE } \geq 18 \\
\text { No vitamin sup- } \\
\text { plements in pre- } \\
\text { vious } 3 \text { months }\end{array}$ & $\begin{array}{l}\text { Design: double-blind random- } \\
\text { ised placebo-controlled } \\
\text { Outcome: secondary } \\
\text { Category: C }\end{array}$ & $\begin{array}{l}\text { T: } 12 \mathrm{mg} \beta \text {-carotene/d, } 400 \mathrm{mg} \\
\text { vitamin E/d and } 500 \mathrm{mg} \\
\text { vitamin C/d } \\
\text { C: placebo (unspecified) } \\
\text { Duration: } 12 \text { months }\end{array}$ \\
\hline Mineral: $\mathrm{Cu}$ & $\begin{array}{l}\text { Kessler et al. } \\
(2008)^{(82)}\end{array}$ & $\begin{array}{l}68 \\
\text { (D/O: 11) }\end{array}$ & $50-80$ & $\begin{array}{l}\text { Alzheimer's dis- } \\
\text { ease, older } \\
\text { adult male, } \\
\text { postmeno- } \\
\text { pausal } \\
\text { female }\end{array}$ & Germany & $\begin{array}{l}\text { MMSE }<25 \\
\text { Clinical diagnosis of } \\
\text { probable Alzhei- } \\
\text { mer's disease } \\
\text { (NINCDS-ADRDA } \\
\text { criteria) } \\
5-10 \mathrm{mg} \text { Donepezil for } \\
2+\text { months }\end{array}$ & $\begin{array}{l}\text { Design: double-blind random- } \\
\text { ised placebo-controlled } \\
\text { Outcome: primary } \\
\text { Category: C }\end{array}$ & $\begin{array}{l}\text { T: } 8 \mathrm{mg} \text { Cu/d } \\
\text { C: placebo (unspecified) } \\
\text { Duration: } 12 \text { months }\end{array}$ \\
\hline
\end{tabular}

Key findings

General:

MMSE (screening)

Memory:

Scanning Memory Sets (NS) Executive function/working memory: Continuous Attention Test (NS) Psychomotor function/processing speed: Four-Choice Reaction Time (NS) Digit-Symbol Substitution (WAIS) $(\dagger P<0.05)$

General:

MMSE (NS) (screening and performance measure)

General:

MMSE (screening)

Telephone Interview for Cognitive Status (screening)

Memory:

Auditory Verbal Learning Test (NS)

Executive function/working memory:

Stroop Colour-Word Test - Abridged (NS) Verbal Fluency Test (NS)

Psychomotor function/processing speed: Digit Symbol Substitution Test (WAIS) $\left({ }^{*} P<0.05\right)$

General:

MMSE (screening)

Memory:

20 Free Recall Task (NS)

Delayed Recognition Memory Task (NS) Logical Reasoning Task (Baddeley) (NS) Executive function/working memory: Categoric Search Task (NS) Focused Attention Task (NS) Repeated-Digits Vigilance Task (NS) Psychomotor function/processing speed: Simple Reaction Time Task (NS)

General:

MMSE (NS) (screening and performance measure) ADAS-Cog (NS) 


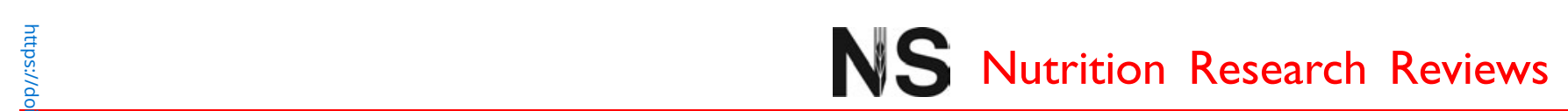

\begin{tabular}{|c|c|c|c|c|c|c|c|c|c|}
\hline Mineral: $\mathrm{Fe}$ & $\begin{array}{l}\text { Murray-Kolb \& } \\
\text { Beard } \\
(2007)^{(81)}\end{array}$ & $\begin{array}{l}152 \\
\text { (D/O: 39) }\end{array}$ & $18-35$ & Adult female & USA & $\begin{array}{l}\text { Three sample groups: } \\
\text { 1. Fe-sufficient } \\
\text { 2. Non-anaemic } \\
\text { Fe-deficient } \\
\text { 3. Anaemic } \\
\text { Fe-deficient }\end{array}$ & $\begin{array}{l}\text { Design: single-blind randomised } \\
\quad \text { placebo-controlled } \\
\text { Outcome: primary } \\
\text { Category: C }\end{array}$ & $\begin{array}{l}\text { T: } 60 \mathrm{mg} \mathrm{Fe} / \mathrm{d} \\
\text { C: placebo (unspecified) } \\
\text { Duration: } 16 \text { weeks }\end{array}$ & 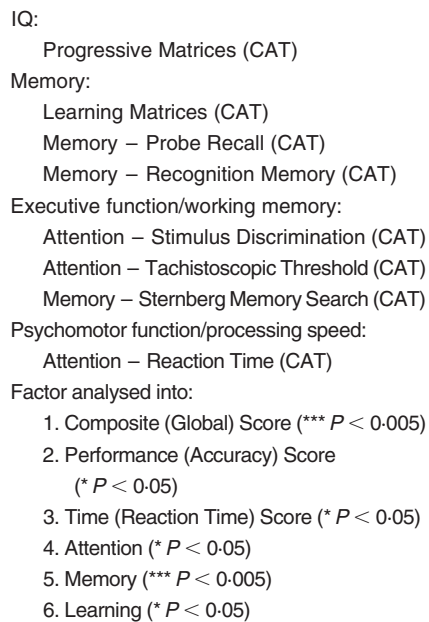 \\
\hline Mineral: $\mathrm{Zn}$ & $\begin{array}{l}\text { Maylor et al. } \\
\quad(2006)^{(80)}\end{array}$ & 387 & $55-87$ & $\begin{array}{l}\text { Adult, older } \\
\text { adult }\end{array}$ & UK & MMSE $\geq 24$ & $\begin{array}{l}\text { Design: double-blind random- } \\
\text { ised placebo-controlled } \\
\text { Outcome: secondary } \\
\text { Category: A }\end{array}$ & $\begin{array}{l}\text { T1: } 30 \mathrm{mg} \mathrm{Zn/d} \\
\text { T2: } 15 \mathrm{mg} \mathrm{Zn/d} \\
\text { C: placebo (unspecified) } \\
\text { Duration: } 6 \text { months }\end{array}$ & $\begin{array}{l}\text { General: } \\
\text { MMSE (screening) } \\
\text { Memory: } \\
\text { Pattern Recognition Memory (CANTAB) (NS) } \\
\text { Spatial Span (CANTAB) (NS) } \\
\text { Executive function/working memory: } \\
\text { Matching to Sample Visual Search } \\
\text { (CANTAB) }{ }^{\star} P<0.05 \text { ) } \\
\text { Spatial Working Memory (CANTAB) } \\
\left.{ }^{{ }^{*} P} P<0.05\right) \\
\text { Psychomotor function/processing speed: } \\
\text { Five-Choice Reaction Time (CANTAB) (NS) } \\
\text { Psychomotor Screening (CANTAB) (NS) } \\
\text { Simple Reaction Time (CANTAB) (NS) }\end{array}$ \\
\hline Flavonoid & $\begin{array}{l}\text { Basaria et al. } \\
(2009)^{(25)}\end{array}$ & mITT: 84 & $46-76$ & $\begin{array}{l}\text { Postmenopau- } \\
\text { sal female }\end{array}$ & USA & n/a & $\begin{array}{l}\text { Design: double-blind random- } \\
\text { ised placebo-controlled } \\
\text { Outcome: secondary } \\
\text { Category: B }\end{array}$ & $\begin{array}{l}\text { T: } 160 \mathrm{mg} \text { total isoflavones/d } \\
\text { C: placebo }(20 \mathrm{~g} \text { whole milk } \\
\text { protein/d }+ \text { same nutrients as } \\
\text { treatment, excluding isoflavones) } \\
\text { Duration: } 12 \text { weeks }\end{array}$ & $\begin{array}{l}\text { IQ: } \\
\text { National Adult Reading Test (performance } \\
\text { measure) (NS) } \\
\text { Executive function/working memory: } \\
\text { Cube Comparisons Test (NS) } \\
\text { Trail-Making Test (HRNTB) (NS) } \\
\text { Verbal Fluency Test (NS) } \\
\text { Psychomotor function/processing speed: } \\
\text { Grooved Pegboard Test (NS) } \\
\text { Identical Pictures Test (NS) }\end{array}$ \\
\hline Flavonoid & $\begin{array}{l}\text { Casini et al. } \\
(2006)^{(84)}\end{array}$ & 78 & About $44-54$ & $\begin{array}{l}\text { Postmenopau- } \\
\text { sal female }\end{array}$ & Italy & n/a & $\begin{array}{l}\text { Design: randomised placebo- } \\
\text { controlled cross-over } \\
\text { Outcome: primary } \\
\text { Category: C }\end{array}$ & $\begin{array}{l}\text { T: } 60 \mathrm{mg} \text { total isoflavones/d } \\
\text { C: placebo (unspecified) } \\
\text { Duration: } 13 \text { months ( } 2 \times 6 \text { month } \\
\text { treatments with } 1 \text { month wash-out) }\end{array}$ & $\begin{array}{l}\text { Memory: } \\
\text { Digit Span Forwards (WAIS) (NS) } \\
\text { Executive function/working memory: } \\
\text { Digit Span Backwards (WAIS) }\left(^{\star} P<0.05\right) \\
\text { Digit Symbol Accuracy (WAIS) }\left(^{\star} P<0.05\right) \\
\text { Visual Scanning Test (NS) } \\
\text { Psychomotor function/processing speed: } \\
\text { Digit Symbol Test (WAIS) }\left(^{\star} P<0.05 \text { ) }\right.\end{array}$ \\
\hline
\end{tabular}




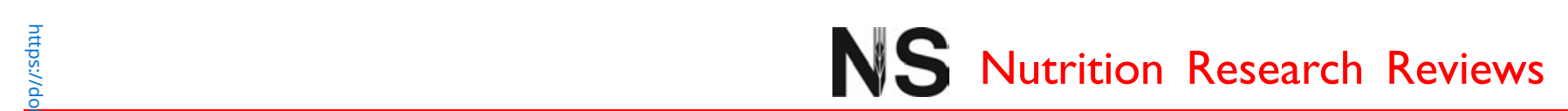

Table 1. Continued

\begin{tabular}{|c|c|c|c|c|c|c|c|c|c|}
\hline Nutrient & Authors & Subjects $(n)$ & Age (years) & $\begin{array}{l}\text { Life stage } \\
\text { and/or sex }\end{array}$ & Setting & $\begin{array}{l}\text { Mental, nutrient } \\
\text { or health status }\end{array}$ & $\begin{array}{l}\text { Design, outcome } \\
\text { status } \ddagger \text { and category§ }\end{array}$ & Intervention, dose and duration & Key findings \\
\hline Flavonoid & $\begin{array}{l}\text { Duffy et al. } \\
\qquad(2003)^{(22)}\end{array}$ & 36 & $50-65$ & $\begin{array}{l}\text { Postmenopau- } \\
\text { sal female }\end{array}$ & UK & $n / a$ & $\begin{array}{l}\text { Design: double-blind random- } \\
\text { ised placebo-controlled } \\
\text { Outcome: primary } \\
\text { Category: B }\end{array}$ & $\begin{array}{l}\text { T: } 60 \mathrm{mg} \text { total isoflavones } / \mathrm{d} \\
\text { C: placebo (colour-matched } \\
\quad \text { lactose) } \\
\text { Duration: } 12 \text { weeks }\end{array}$ & $\begin{array}{l}\text { IQ: } \\
\text { National Adult Reading Test (to check } \\
\text { baseline differences) } \\
\text { Memory: } \\
\text { Common Objects Recall Test }\left({ }^{\star} P<0.05\right) \\
\text { Delayed Matching To Sample Test } \\
\text { (CANTAB) (NS) } \\
\text { Short Story Immediate Recall (NS) } \\
\text { Executive function/working memory: } \\
\text { Category Generation (NS) } \\
\text { IDED Rule Learning and Reversal } \\
\text { (CANTAB) ( } P<0.05) \\
\text { Paced Auditory Serial Addition Test } \\
\left(^{*} P<0.05\right) \\
\text { Stockings of Cambridge (CANTAB) } \\
\left(^{*} P<0.05\right)\end{array}$ \\
\hline Flavonoid & $\begin{array}{l}\text { Elsabagh et al. } \\
\quad(2005)^{(26)} \\
\quad \text { Experiment } 2\end{array}$ & 40 & $18-26$ & Young adult & UK & $n / a$ & $\begin{array}{l}\text { Design: double-blind random- } \\
\text { ised placebo-controlled } \\
\text { Outcome: primary } \\
\text { Category: C }\end{array}$ & $\begin{array}{l}\text { T: } 120 \mathrm{mg} \text { Ginkgo biloba/d (Ginkyo, } \\
\text { Lichtwer Pharma - } 25 \% \text { total } \\
\text { flavonoids, } 6 \% \text { total terpenoids) } \\
\text { C: placebo (unspecified) } \\
\text { Duration: } 6 \text { weeks }\end{array}$ & $\begin{array}{l}\text { Memory: } \\
\text { Delayed Recall of Words and Pictures } \\
\text { (CANTAB) (NS) } \\
\text { Pattern Recognition Memory (CANTAB) (NS) } \\
\text { Spatial Recognition Memory (CANTAB) (NS) } \\
\text { Executive function/working memory: } \\
\text { IDED Rule Learning and Reversal } \\
\text { (CANTAB) (NS) } \\
\text { Paced Auditory Serial Addition Test (NS) } \\
\text { Spatial Working Memory (CANTAB) (NS) } \\
\text { Stockings of Cambridge (CANTAB) (NS) }\end{array}$ \\
\hline Flavonoid & $\begin{array}{l}\text { File et al. } \\
\qquad(2001)^{(21)}\end{array}$ & 27 & About 22-30 & Young adult & UK & $n / a$ & $\begin{array}{l}\text { Design: randomised controlled } \\
\text { Outcome: primary } \\
\text { Category: B }\end{array}$ & $\begin{array}{l}\text { T: } 100 \mathrm{mg} \text { total isoflavones/d ('high') } \\
\text { C: } 0.5 \mathrm{mg} \text { total isoflavones } / \mathrm{d} \text { ('low', } \\
\text { i.e. control) } \\
\text { Duration: } 10 \text { weeks }\end{array}$ & 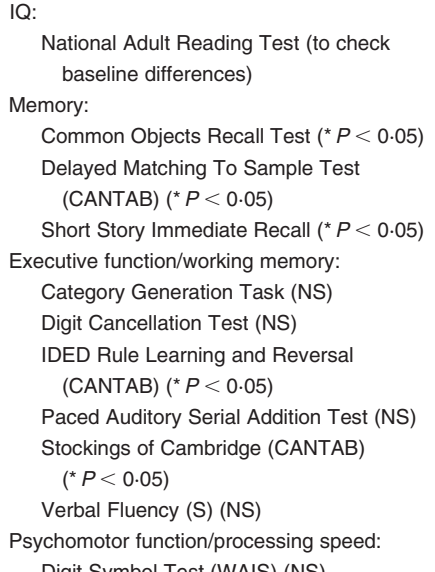 \\
\hline
\end{tabular}




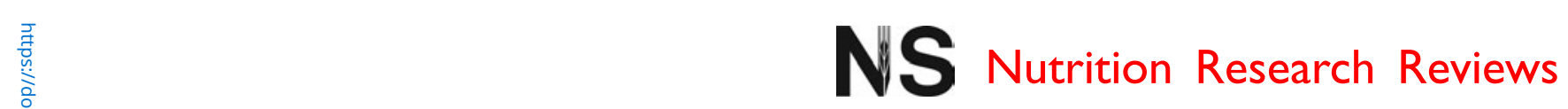

\begin{tabular}{|c|c|c|c|c|c|c|}
\hline Flavonoid & $\begin{array}{l}\text { File et al. } \\
\quad(2005)^{(19)}\end{array}$ & 50 & 51-66 & $\begin{array}{l}\text { Postmenopau- } \\
\text { sal female }\end{array}$ & UK & n/a \\
\hline Flavonoid & $\begin{array}{l}\text { Fournier et al. } \\
(2007)^{(27)}\end{array}$ & 79 & $48-65$ & $\begin{array}{l}\text { Postmenopau- } \\
\text { sal female }\end{array}$ & USA & $\mathrm{n} / \mathrm{a}$ \\
\hline Flavonoid & $\begin{array}{l}\text { Francis et al. } \\
(2006)^{(87)}\end{array}$ & 16 & $18-30$ & Adult female & UK & n/a \\
\hline Flavonoid & $\begin{array}{l}\text { Gleason et al. } \\
\quad(2009)^{(20)}\end{array}$ & 30 & $62-89$ & $\begin{array}{l}\text { Older adult } \\
\text { male, post- } \\
\text { menopausal } \\
\text { female }\end{array}$ & USA & MMSE $\geq 27$ \\
\hline
\end{tabular}

$\begin{array}{cl}\begin{array}{c}\text { Design: double-blind random- } \\ \text { ised placebo-controlled - }\end{array} & \text { T: } 60 \mathrm{mg} \text { total isoflavones/d } \\ \text { parallel groups } & \text { C: placebo (unspecified) } \\ \text { Outcome: primary } & \\ \text { Curation: } 6 \text { weeks }\end{array}$

Category: C

Design: double blind randomised placebo-controlled Outcome: primary Category: C

Design: double-blind randomised placebo-controlled cross-over

Outcome: primary

Category: B

Design: double-blind random-

menopaus

female
Outcome: secondary

Category: C
T1: $70 \mathrm{mg}$ total isoflavones/d (supplement + cows' milk) (soya milk + cows' milk) C: placebo (cows' milk) Duration: 16 weeks

T: 172 mg ('high') cocoa flavanols/d C: $13 \mathrm{mg}$ ('low') cocoa flavanols/d Duration: $24+d(2 \times 5 d$ treatments with at least $14 \mathrm{~d}$ wash-out)

T: $100 \mathrm{mg}$ total isoflavones/d C: placebo (maltodextrin + caramel

$$
\text { food colour) }
$$

Duration: 6 months T2: $72 \mathrm{mg}$ total isoflavones/d
IQ:

National Adult Reading Test (to check baseline differences)

Memory:

Common Objects Recall Test $\left.{ }^{\star} P<0.05\right)$ Delayed Matching To Sample Test (CANTAB) (NS)

Short Story Immediate $\left({ }^{*} P<0.05\right)$ and Short Story Immediate
Delayed Recall (NS)

Executive function/working memory: Category Generation Task (NS) IDED Rule Learning and Reversal (CANTAB) $\left({ }^{*} P<0.05\right)$ Paced Auditory Serial Addition Test (NS) Stockings of Cambridge (CANTAB) $\left({ }^{*} P<0.05\right)$

Memory:

Corsi Block-Tapping Task (NS) Digit Span Forwards (WAIS) (NS) Pattern Recognition Task (NS) Benton Visual Retention Test (NS)

Executive function/working memory Colour Matching Task (NS) Digit Ordering Task $(\dagger P<0.05)$ Stroop Colour-Word Task (NS) Executive function/working memory: fMRI BOLD signal $\left(^{*} P<0.05\right)$ Letter-Digit Pairs Task (NS)

General:

MMSE (screening)

Memory:

Boston Naming Test (NS)

Buschke Selective Reminding Test (NS) Paragraph Recall Test (WMS) (NS) Visual Spatial Learning Test $\left({ }^{*} P<0.05\right)$

Executive function/working memory: Category Fluency Test (Animals) $\left({ }^{*} P<0.05\right)$

Controlled Oral Word Association Test (NS) Maze-Tracing Task (NS)

Rey-Osterrieth Complex Figure Test $\left({ }^{*} P<0.05\right)$

Stroop Colour-Word Task ( $P<0.05)$ Trail-Making Test (HRNTB) $(\dagger P<0.05)$

Psychomotor function/processing speed:

Grooved Pegboard $\left({ }^{*} P<0.05\right)$ Rey Complex Figure Test copy $\left({ }^{*} P<0.05\right)$ 


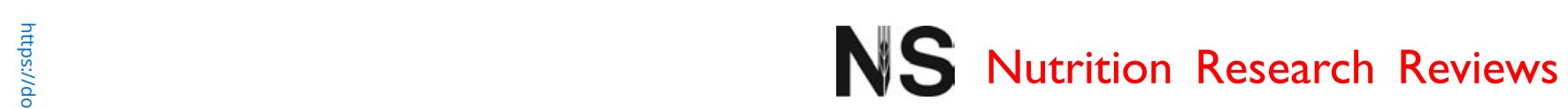

\begin{tabular}{|c|c|c|c|c|c|c|}
\hline Flavonoid & $\begin{array}{l}\text { Kritz-Silverstein } \\
\quad \text { et al. } \\
\quad(2003)^{(86)}\end{array}$ & 56 & $55-74$ & $\begin{array}{l}\text { Postmenopau- } \\
\text { sal female }\end{array}$ & USA & n/a \\
\hline Flavonoid & $\begin{array}{l}\text { Le Bars et al. } \\
\qquad(2002)^{(23)}\end{array}$ & 236 & $45-90$ & $\begin{array}{l}\text { Alzheimer's dis- } \\
\text { ease, older } \\
\text { adult }\end{array}$ & USA & $\begin{array}{l}\text { MMSE }>23 \\
M M S E<24 \\
M M S E<15\end{array}$ \\
\hline Flavonoid & $\begin{array}{l}\text { Mix \& Crews } \\
\quad(2000)^{(88)}\end{array}$ & 48 & $55-86$ & Older adult & USA & MMSE $>23$ \\
\hline Flavonoid & $\begin{array}{l}\text { Ryan et al. } \\
(2008)^{(90)}\end{array}$ & 101 & $60-85$ & Older adult & Australia & $n / a$ \\
\hline
\end{tabular}

Design: double-blind random ised placebo-controlled Outcome: primary

Category: B

Design: double-blind randomised placebo-controlled Outcome: primary Category: B

Design: double-blind randomised placebo-controlled Outcome: primary Category: C

Design: double-blind randomised placebo-controlled Outcome: primary Category: B

\section{T: $110 \mathrm{mg}$ total soya isoflavones/d C: placebo (unspecified)} Duration: 6 months

T: $120 \mathrm{mg}$ G. biloba extract EG $761 / d$ ( $24 \%$ total flavonoids, $6 \%$ total terpenoids) C: placebo (unspecified) Duration: 12 months

T: $180 \mathrm{mg}$ G. biloba extract EGb $761 / \mathrm{d}$ (24\% total flavonoids,

$$
6 \% \text { total terpenoids) }
$$

C: placebo (unspecified) Duration: 6 weeks

T: $150 \mathrm{mg}$ total flavonoids/d (French maritime pine extract 'Pycnogenol®') C: placebo (unspecified) Duration: 3 months
General:

MMSE (NS) (to check baseline differences) Memory:

Logical Memory 1 and 2 Tests (NS) Executive function/working memory: Category Fluency $\left({ }^{*} P<0.05\right)$ Trail-Making Test (HRNTB) (NS) General: MMSE (screening) ADAS-Cog $\left({ }^{*} P<0.05\right)$

General: MMSE (screening)

Memory:

Logical Memory 1 and 2 Tests (WMS) (NS) Visual Memory 1 and 2 Tests (WMS) (NS) Executive function/working memory: Stroop Colour-Word Task (NS) Trail-Making Test (HRNTB) (NS) Psychomotor function/processing speed: Stroop Colour Naming Task $\left({ }^{\star} P<0.05\right)$ Stroop Word Naming Task (NS) Trail-Making Test - Part A (HRNTB) (NS) Memory:

Word Recognition (CDR) (NS) Executive function/working memory Digit Vigilance (CDR) (NS) Numeric Working Memory (CDR) (NS) Spatial Working Memory (CDR) $\left({ }^{*} P<0.05\right)$

Psychomotor function:

Choice Reaction Time (CDR) (NS) Simple Reaction Time (CDR) (NS) Factor analysed as:

1. Power of Attention (NS):

Digit Vigilance (NS)

Simple Reaction Time (NS) Choice Reaction Time (NS)

2. Quality of Working Memory $\left({ }^{* *} P<0.01\right.$ ): Spatial Working Memory ( ${ }^{*} P<0.05$ ) Numeric Working Memory (NS) 


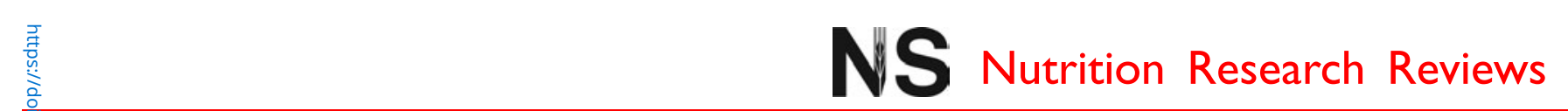

Table 1. Continued

\begin{tabular}{|c|c|c|c|c|c|c|c|c|}
\hline Flavonoid & $\begin{array}{l}\text { Santos et al. } \\
\quad(2003)^{(91)}\end{array}$ & 48 & $60-70$ & $\begin{array}{c}\text { Older adult } \\
\text { male }\end{array}$ & Brazil & MMSE $>23$ & $\begin{array}{l}\text { Design: double-blind random- } \\
\text { ised placebo-controlled } \\
\text { Outcome: primary } \\
\text { Category: C }\end{array}$ & $\begin{array}{l}\text { T: } 80 \mathrm{mg} \text { G. biloba/d (Magister } \\
\quad \text { Medicamentos - } 24 \% \text { flavo- } \\
\quad \text { noids, } 6 \% \text { terpenoids) } \\
\text { C: placebo (unspecified) } \\
\text { Duration: } 8 \text { months }\end{array}$ \\
\hline
\end{tabular}

Key findings

IQ:

Comprehension Test (WAIS)

$\left({ }^{\star \star *} P<0.005\right)$

Information Test (WAIS)

Information Test (WMS)

Vocabulary Test (WAIS) $\left.{ }^{\star *} P<0.01\right)$

General:

MMSE (screening)

Orientation Test (WMS)

Memory

Corsi Block-Tapping Test $\left.{ }^{(* *} P<0.005\right)$

Digit Span Forward (WAIS)

Logical Memory Test (WMS)

Similarities Test (WAIS) $\left.{ }^{* \star \star} P<0.005\right)$

Verbal Free Recall Test (NS)

Verbal Paired Associates Test (WMS)

$\left({ }^{* * *} P<0.005\right)$

Executive function/working memory:

Arithmetic Test (WAIS) $\left.{ }^{\star * * *} P<0.005\right)$

Block Design Test (WAIS) $\left.{ }^{* *} P<0.01\right)$

Digit Span Backward (WAIS)

Mental Control Test (WMS) $\left.{ }^{* * *} P<0.005\right)$

Object Assembly Test (WAIS)

$\left({ }^{* \star *} P<0.005\right)$

Picture Arrangement Test (WAIS)

Picture Completion Test (WAIS)

Rey-Osterrieth Complex Figure Test

$\left({ }^{* *} P<0.01\right.$ - delayed $)$

Concentrated Attention Test (TP)

$\left.{ }^{* \star \star} P<0.005\right)$

Wisconsin Card Sorting Test (NS)

Psychomotor function/processing speed: Digit Symbol Substitution Task (WAIS) $\left({ }^{* \star *} P<0.005\right)$

Design: double-blind randomised placebo-controlled Outcome: primary Category: C
T: $120 \mathrm{mg}$ G. biloba/d (Blackmore's Ginkgo Biloba Forte $-24 \%$ total flavonoids, $6 \%$ total terpenoids) C: placebo (unspecified) Duration: 1 month
Digit Span Forward (WAIS) (NS) Rey Auditory-Verbal Learning Test $\left({ }^{* *} P<0.01\right)$

Executive function/working memory: Digit Span Backward (WAIS) $\left(^{*} P<0.05\right)$ Trail-Making Test (HRNTB) (NS) Working Memory (CBT) (NS)

Psychomotor function/processing speed: Digit Symbol Substitution Task (WAIS $\left({ }^{*} P<0.05\right)$

Inspection Time Test (NS)

Simple Reaction Time (CBT) (NS) Speed of Comprehension Test (SCOLP) (NS) 


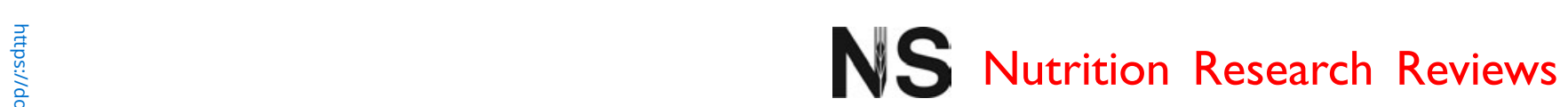

\begin{tabular}{|c|c|c|c|c|c|c|c|c|c|}
\hline Flavonoid & $\begin{array}{l}\text { van Dongen et al. } \\
(2000)^{(29)}\end{array}$ & ITT: 214 & $50+$ & $\begin{array}{l}\text { Dementia, older } \\
\text { adult }\end{array}$ & $\begin{array}{l}\text { The Nether- } \\
\text { lands }\end{array}$ & MMSE 7-29 & $\begin{array}{l}\text { Design: double-blind random- } \\
\text { ised placebo-controlled } \\
\text { Outcome: primary } \\
\text { Category: B }\end{array}$ & $\begin{array}{l}\text { T1: } 240 \mathrm{mg} \text { G. biloba extract EGb } \\
761 / \mathrm{d} \\
\text { T2: } 160 \mathrm{mg} \text { G. biloba extract EGb } \\
\quad 761 / \mathrm{d} \\
\text { C: placebo (unspecified) } \\
\text { Duration: } 24 \text { weeks }\end{array}$ & $\begin{array}{l}\text { General: } \\
\text { MMSE (screening) } \\
\text { Memory: } \\
\text { Digit Span Forward (WAIS) (NS) } \\
\text { 8-Word List (NS) } \\
\text { Executive function/working memory: } \\
\text { Digit Span Backward (WAIS) (NS) } \\
\text { Psychomotor function/processing speed: } \\
\text { Trail-Making Test - Part A (NS) }\end{array}$ \\
\hline Flavonoid & $\begin{array}{l}\text { Woo et al. } \\
(2003)^{(89)}\end{array}$ & 127 & $50-65$ & $\begin{array}{l}\text { Postmenopau- } \\
\text { sal female }\end{array}$ & Hong Kong & $n / a$ & $\begin{array}{l}\text { Design: randomised controlled } \\
\text { Outcome: secondary } \\
\text { Category: C }\end{array}$ & $\begin{array}{l}\text { T1: } 100 \mathrm{mg} \text { total isoflavones/d (from } \\
\quad \text { Pueraria lobata) } \\
\text { T2: HRT } \\
\text { C: no treatment } \\
\text { Duration: } 3 \text { months }\end{array}$ & $\begin{array}{l}\text { General: } \\
\left.\text { MMSE ( }{ }^{\star} P<0.05\right) \\
\text { Memory: } \\
\quad \text { Hong Kong List-Learning Test (NS) } \\
\text { Executive function/working memory: } \\
\text { Boston Naming Test (NS) } \\
\text { Trail-Making Test, also referred to as } \\
\quad \text { Figure Trail } 1 \text { and } 2\left({ }^{\star} P<0.05\right) \\
\text { Psychomotor function: } \\
\text { Digit Symbol Test (cited in Results } \\
\text { section only) }\left(^{\star} P<0.05\right) \\
\text { Finger-Tapping Test (NS) }\end{array}$ \\
\hline
\end{tabular}

MCI, mild cognitive impairment; DSM, Diagnostic and Statistical Manual of Mental Disorders; MMSE, Mini-Mental State Examination; T, treatment; C, control; ADAS-Cog, Cognitive element of the Alzheimer's Disease Assessment Scale; n/a, not applicable; IQ, intelligence quotient; ITT, intention-to-treat; D/O, dropped out; RDA, recommended daily allowance; WAIS, Wechsler Adult Intelligence Scale (all versions); HRNTB, Halstead-Reitan Neuropsychological Test Battery; CAMCOG, Cambridge Cognitive Examination; WMS, Wechsler Memory Scale (all versions); mITT, modified intention-to-treat; TICS, Telephone Interview for Cognitive Status; NINDS-ADRDA, National Institute of Neurological Disorders and Stroke and Alzheimer's Disease and Related Disorders Association; CAT, Cognitive Abilities Test; CANTAB, Cambridge Neuropsychological Test Automated Battery; IDED, Intra Dimensional/Extra Dimensional Set Shifting Task; fMRI, functional magnetic resonance imaging; BOLD, blood oxygenation level-dependent; CDR, cognitive drug research; TP, Toulouse-Pieron Test; CBT, Cognometer Battery of Tests; SCOLP, Speed and Capacity of Language-Processing Test; HRT, hormone replacement therapy.

${ }^{*}$ Mean treatment group scores were significantly better than those of the control group: ${ }^{*} P<0.05,{ }^{* \star} P<0.01,{ }^{* \star *} P<0.005$.

† Mean treatment group scores were significantly worse than those of the control group: $\dagger P<0.05$, $\uparrow \dagger P<0.01$, †† $P<0.005$.

$¥$ Study designed with cognitive function as 'primary' or 'secondary' target outcome measure.

$\S$ Study graded for quality: category A, highest quality, no bias; category B, medium quality, some bias but results are deemed valid; category C, poor quality, significant bias that may invalidate the results. $\| 1 \mathrm{IU}$ vitamin $\mathrm{A}=0.3 \mu \mathrm{g}$ retinol or $0.6 \mu \mathrm{g} \beta$-carotene. 
with multiple populations and settings; none was specifically designed with reference to micronutrient or phytochemical interventions. Revealingly, in the remaining eighteen studies $(20,24-26,66,67,69,70,72,74,75,78,82,84,86,89,91,92)$, no rationale for task choice was given, although four of these included dementia patients, so task selection was naturally restricted to measures appropriate to these populations $(66,70,74,82)$.

\section{Range of cognitive measures used}

Across the thirty-nine RCT under investigation, 121 cognitive tasks were identified (see Table 2). After an analysis of the primary neuropsychological focus for each measure, it was calculated that thirty-seven memory tasks (for example, episodic, semantic and short-term), twenty-six executive function tasks, fourteen working memory tasks, nineteen psychomotor processing speed tasks, nine general or 'global' tasks, thirteen intelligence quotient (IQ) tasks (mostly to measure baseline between-group differences), two motor function tasks and one perception measure had been employed (see Table 2).

Generally, there was little correspondence in measures between studies, with occasional notable exceptions. For instance, researchers from King's College London ${ }^{(19,21,22)}$ used the same seven executive function and memory tasks in their flavonoid intervention studies on older populations as had previously been used in a group of 22-to 30-year-old subjects $^{(19)}$. Two tasks which were non-significant in the earlier study were excluded. While they found the Common Objects Recall Test and the Cambridge Neuropsychological Test Automated Battery (CANTAB) Intra Dimensional/ Extra Dimensional Set Shifting Task Rule Learning and Reversal tests to be sensitive to flavonoid treatment in all three studies, these tasks appear to have been rarely employed elsewhere (see Table 2).

\section{Executive function}

Among the executive function tasks, one measure was primarily categorised as 'focused attention' and five as 'sustained attention'. Four measured 'switching' or 'shifting', and one measured 'inhibition'. None focused specifically on 'updating', although two tasks described here as measuring the more generalised 'frontal function' may include varying degrees of 'updating' as well as 'switching' and 'inhibition'. In addition, there were five measures of 'verbal fluency', four 'visual search', two 'decision-making' and one 'planning' task (see Table 2).

Verbal fluency tasks were used in sixteen studies, and some positive effects were shown in four: flavonoids, B vitamins and multivitamins and minerals ${ }^{(20,68,79,86)}$. Verbal fluency tasks included category generation, where participants are asked to name as many category members as possible (for example, animals, transportation, etc), and initial-letter verbal fluency, where participants generate words beginning with a particular letter in a given time (for example, F, A or S). Category fluency tasks, which are also thought to involve an element of semantic memory ${ }^{(62)}$, were significant in the two flavonoid studies $(20,86)$ and the multivitamin and minerals study ${ }^{(68)}$, although
Wouters-Wesseling et al. ${ }^{(68)}$ reported significant baseline differences on this task, suggesting potential randomisation issues in this study. The less semantically demanding initial-letter fluency tasks were generally non-significant, with the exception of Bryan et al. ${ }^{(79)}$ who found significantly better fluency scores for vitamin $\mathrm{B}_{6}$ and placebo than for folate or vitamin $\mathrm{B}_{12}$.

The Stroop Colour-Word Task, which measures inhibition, was used in seven studies, and showed positive effects of flavonoid treatment in two ${ }^{(20,88)}$. Conspicuously, the Trail-Making Task, which featured in thirteen studies $^{(20,24,25,28,29,71,72,79,85,86,88,89,92)}$ showed only a negative effect of treatment for vitamin $\mathrm{B}^{(72)}$ and flavonoids ${ }^{(20)}$. Although a common measure of 'switching', it is possible that the pen and paper nature of the Trail-Making Task limits its sensitivity. However, none of the four 'switching' tasks reviewed here has shown a positive treatment effect, perhaps suggestive of a more general limiting condition.

The Intra Dimensional/Extra Dimensional Set Shifting Task Rule Learning and Reversal task which measures frontal function, involving aspects of 'switching' or 'shifting', 'updating' and 'inhibition', and the Stockings of Cambridge planning task showed some sensitivity to flavonoid treatment for both young ${ }^{(19)}$ and older ${ }^{(21,22)}$ adults, for doses of 60-100 mg total isoflavones per d, over periods ranging from 6 to 12 weeks (see Tables 1 and 2). However, both tests failed to show any effect in a 6-week $G$. biloba trial in young adults ${ }^{(26)}$.

\section{Working memory}

Eight measures of visuospatial or spatial working memory were identified, along with three numerical measures, one verbal measure, and one test of working memory span (for example, the Digit Span Backwards task which measures working memory capacity), which was used in nine studies (see Table 2). The eight visuospatial measures, used in eleven different studies, showed significant effects in four studies, with respect to flavonoid treatments ${ }^{(20,90,91)}$ and $\mathrm{Zn}^{(80)}$, although Santos et al. ${ }^{(91)}(n 48)$ and Gleason et al. ${ }^{(20)}$ ( $n$ 30) had relatively small sample sizes, and the randomisation process adopted by Santos et al. ${ }^{(91)}$ may not have been reliable as they reported baseline differences in IQ between groups.

Two computerised tasks described as measuring 'spatial working memory' appeared sensitive to $\mathrm{Zn}^{(80)}$ and flavonoid ${ }^{(90)}$ treatment. Encouragingly, while they were described as measuring the same cognitive function, the tasks themselves varied considerably, suggesting that the observed effects were specific to domain rather than task. For instance, the Zn study Cambridge Neuropsychological Test Automated Battery (CANTAB) task required participants to search for, and remember, the locations of blue tokens inside a set of red boxes. In the Ryan et al. ${ }^{(90)}$ study, participants were shown a picture of a house and were asked to memorise the locations of nine lit windows; subsequently, they were shown individually lit windows and asked to decide if they had been lit earlier. The Rey-Osterrieth Complex Figure Test, used in four studies, showed significant positive treatment effects in two ${ }^{(20,91)}$. 


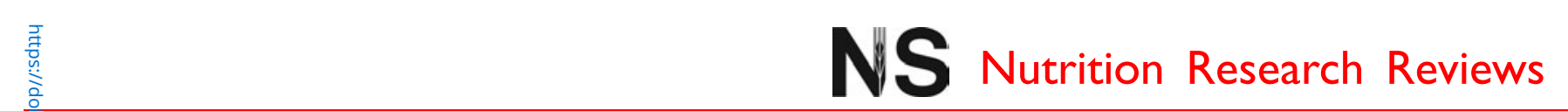

Table 2. Continued

\begin{tabular}{|c|c|c|c|c|c|c|c|c|c|c|c|c|c|c|c|c|c|c|c|c|c|c|c|c|c|c|c|c|}
\hline \multirow[b]{2}{*}{$\begin{array}{l}\text { Primary } \\
\text { neuropsychological } \\
\text { focus }\end{array}$} & \multirow[b]{2}{*}{ Task and $\mathrm{RCT}$ reference } & \multirow[b]{2}{*}{$\begin{array}{l}\text { Significant positive or } \\
\text { negative treatment effects }\end{array}$} & \multicolumn{9}{|c|}{ Executive function } & \multicolumn{12}{|c|}{ Memory } & \multicolumn{2}{|c|}{ Motor } & \multicolumn{3}{|c|}{ IQ } \\
\hline & & & 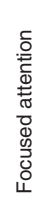 & 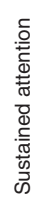 & 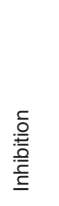 & 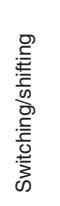 & 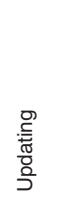 & 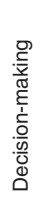 & 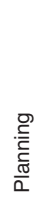 & 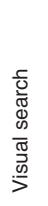 & 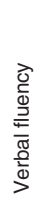 & 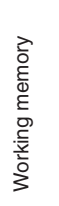 & 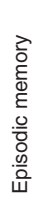 & 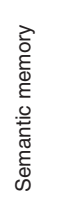 & 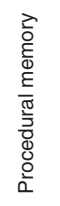 & 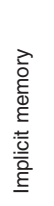 & 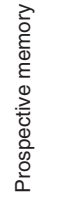 & 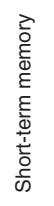 & 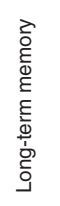 & 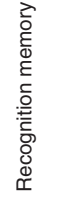 & 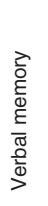 & 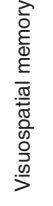 & 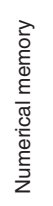 & 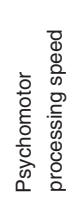 & 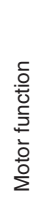 & 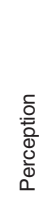 & 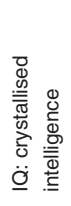 & 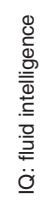 \\
\hline Exec Fn: inhibition & $\begin{array}{l}\text { Stroop Colour-Word } \\
\text { Task }^{(20,27,71,75,77,79,88)}\end{array}$ & $\begin{array}{l}\text { Positive: flavonoid } \\
\text { Negative: flavonoid } \\
\text { (20) }\end{array}$ & & & $\mathrm{Y}$ & & & & & & & Y & & & & & & & & & & & & $\mathrm{Y}$ & & & & \\
\hline Exec Fn: planning & $\begin{array}{l}\text { Stockings of Cambridge } \\
\text { (CANTAB) }^{(19,21,22,26)}\end{array}$ & Positive: flavonoid(19,21,22) & & & & & & & Y & & & $\mathrm{Y}$ & & & & & & & & & & & & & & & & \\
\hline $\begin{array}{l}\text { Exec Fn: sustained } \\
\text { attention }\end{array}$ & Continuous Attention Test ${ }^{(73)}$ & - & & Y & & & & & & & & Y & & & & & & & & & & & & $\mathrm{Y}$ & & & & \\
\hline $\begin{array}{l}\text { Exec Fn: sustained } \\
\text { attention }\end{array}$ & $\begin{array}{l}\text { Digit Vigilance Test/Digit } \\
\quad \text { Vigilance Test }(\mathrm{CDR})^{(24,90)}\end{array}$ & - & & $\mathrm{Y}$ & & & & & & & & $\mathrm{Y}$ & & & & & & & & & & & & $\mathrm{Y}$ & & & & \\
\hline $\begin{array}{l}\text { Exec Fn: sustained } \\
\text { attention }\end{array}$ & Mental Control Task ${ }^{(91)}$ & Positive: flavonoid ${ }^{(91)}$ & & $\mathrm{Y}$ & & & & & & & & $\mathrm{Y}$ & & & & & & & & & & & & $\mathrm{Y}$ & & & & \\
\hline $\begin{array}{l}\text { Exec Fn: sustained } \\
\text { attention }\end{array}$ & $\begin{array}{l}\text { Paced Auditory Serial Addition } \\
\text { Test }^{(19,21,22,26)}\end{array}$ & Positive: flavonoid ${ }^{(22)}$ & & $\mathrm{Y}$ & & & & & & & & $\mathrm{Y}$ & & & & & & & & & & & & $\mathrm{Y}$ & & & & \\
\hline $\begin{array}{l}\text { Exec Fn: sustained } \\
\text { attention }\end{array}$ & Repeated-Digits Vigilance Task ${ }^{(83)}$ & - & & Y & & & & & & & & Y & & & & & & & & & & & & Y & & & & \\
\hline Exec Fn: switching/shifting & Concept Shifting Test ${ }^{(77)}$ & - & & & & $\mathrm{Y}$ & & & & & & $\mathrm{Y}$ & & & & & & & & & & & & $\mathrm{Y}$ & & & & \\
\hline Exec Fn: switching/shifting & Letter-Digit Pairs Task ${ }^{(87)}$ & - & & & & $\mathrm{Y}$ & & & & & & $\mathrm{Y}$ & & & & & & & & & & & & Y & & & & \\
\hline Exec Fn: switching/shiftting & Maze-Tracing Task ${ }^{(20)}$ & - & & & & $\mathrm{Y}$ & & & & & & $\mathrm{Y}$ & & & & & & & & & & & & Y & & & & \\
\hline Exec Fn: switching/shifting & $\begin{array}{l}\text { Trail-Making Test } \\
\text { (HRNTB) }^{(20,24,25,28,29,71,72,79,85,86,88,89,92)}\end{array}$ & $\begin{array}{l}\text { Negative: } B \text { vitamins } s^{(72)} \\
\text { Negative: } \text { flavonoid }^{(20)}\end{array}$ & & & & Y & & & & $\mathrm{Y}$ & & Y & & & & & & & & & & & & Y & & & & \\
\hline Exec Fn: verbal fluency & $\begin{array}{l}\text { Category Fluency Tests: } \\
\text { Category Fluency Test (Animals, } \\
\text { Transportation), Category Word } \\
\text { Fluency Test (Animals, } \\
\text { Professions) } \\
\text { (20,24,68,71,72,85,86) }^{-}\end{array}$ & $\begin{array}{l}\text { Positive: flavonoid }{ }^{(20,86)} \\
\text { Positive: multivitamins/ } \\
\text { multiminerals }\end{array}$ & & & & & & & & & $\mathrm{Y}$ & Y & & $\mathrm{Y}$ & & & & & & & & & & & & & & \\
\hline Exec Fn: verbal fluency & Category Generation Task ${ }^{(19,21,22)}$ & - & & & & & & & & & $\mathrm{Y}$ & $\mathrm{Y}$ & & $\mathrm{Y}$ & & & & & & & & & & & & & & \\
\hline Exec Fn: verbal fluency & $\begin{array}{l}\text { Controlled Oral Word Association } \\
\text { Test }(20,72,85)\end{array}$ & - & & & & & & & & & Y & Y & & & & & & & & & & & & & & & & \\
\hline Exec Fn: verbal fluency & Uses for Common Objects ${ }^{(79)}$ & - & & & & & & & & & $\mathrm{Y}$ & $\mathrm{Y}$ & & $\mathrm{Y}$ & & & & & & & & & & & & & & \\
\hline Exec Fn: verbal fluency & $\begin{array}{l}\text { Verbal Fluency Tests: Verbal } \\
\text { Fluency Test (Initial Letter, } \\
\text { Excluded Letter) }{ }^{(21,25,28,6,7,71,75,77,79)}\end{array}$ & Negative: B vitamins ${ }^{(79)}$ & & & & & & & & & Y & Y & & & & & & & & & & & & & & & & \\
\hline Exec Fn: visual search & Digit Cancellation Test ${ }^{(21)}$ & - & & & & & & & & $\mathrm{Y}$ & & $\mathrm{Y}$ & & & & & & & & & & & & $\mathrm{Y}$ & & & & \\
\hline Exec Fn: visual search & $\begin{array}{l}\text { Matching to Sample Visual Search } \\
\text { (CANTAB) })^{(80)}\end{array}$ & Positive: $\mathrm{Zn}^{(80)}$ & & & & & & & & Y & & Y & & & & & & & & & & & & Y & & & & \\
\hline Exec Fn: visual search & $\begin{array}{l}\text { Sternberg Memory Search Task } \\
\text { (CAT) }\end{array}$ & - & & & & & & & & $\mathrm{Y}$ & & $\mathrm{Y}$ & & & & & & & & & & & & $\mathrm{Y}$ & & & & \\
\hline Exec Fn: visual search & Visual Scanning Test ${ }^{(84)}$ & - & & & & & & & & $\mathrm{Y}$ & & $\mathrm{Y}$ & & & & & & & & & & & & Y & & & & \\
\hline Working memory & Working Memory $(\mathrm{CBT})^{(92)}$ & - & & & & & & & & & & $\mathrm{Y}$ & & & & & & & & & & & & & & & & \\
\hline $\begin{array}{l}\text { Working memory: } \\
\text { numerical }\end{array}$ & Arithmetic Test (WAIS/WMS) ${ }^{(85,91)}$ & - & & & & & & & & & & Y & & & & & & & & & & & $\mathrm{Y}$ & & & & & \\
\hline $\begin{array}{l}\text { Working memory: } \\
\text { numerical }\end{array}$ & Numeric Working Memory $(\mathrm{CDR})^{(90)}$ & - & & & & & & & & & & $\mathrm{Y}$ & & & & & & & & & & & Y & & & & & \\
\hline $\begin{array}{l}\text { Working memory: } \\
\text { numerical }\end{array}$ & Scanning Memory Sets ${ }^{(73)}$ & - & & & & & & & & & & $\mathrm{Y}$ & & & & & & & & & & & $\mathrm{Y}$ & & & & & \\
\hline
\end{tabular}




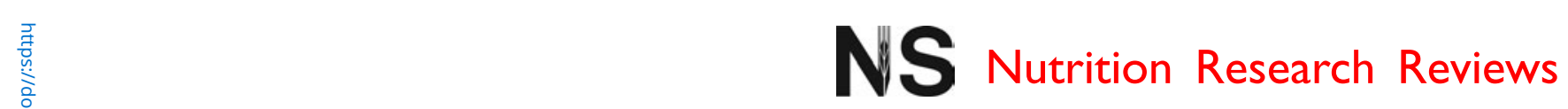

\begin{tabular}{|c|c|c|}
\hline Working memory: span & $\begin{array}{l}\text { Digit Span Backward } \\
\text { (WAIS) })^{(24,28,29,71,78,84,91,92)}\end{array}$ & Positive: flavonoid ${ }^{(84,92)}$ \\
\hline Working memory: verbal & Digit Ordering $^{(27)}$ & Negative: flavonoid ${ }^{(27)}$ \\
\hline $\begin{array}{l}\text { Working memory: visual/ } \\
\text { visuospatial }\end{array}$ & Block Design Test (WAIS) $)^{(78,85,91)}$ & Positive: flavonoid $^{(91)}$ \\
\hline $\begin{array}{l}\text { Working memory: visual/ } \\
\text { visuospatial }\end{array}$ & Colour Matching Task ${ }^{(27)}$ & - \\
\hline $\begin{array}{l}\text { Working memory: visual/ } \\
\text { visuospatial }\end{array}$ & Cube Comparisons Test( ${ }^{(25)}$ & - \\
\hline $\begin{array}{l}\text { Working memory: visual/ } \\
\text { visuospatial }\end{array}$ & Object Assembly Test (WAIS) ${ }^{(91)}$ & Positive: flavonoid ${ }^{(91)}$ \\
\hline $\begin{array}{l}\text { Working memory: visual/ } \\
\text { visuospatial }\end{array}$ & Picture Completion Test (WAIS) ${ }^{(91)}$ & 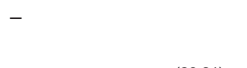 \\
\hline $\begin{array}{l}\text { Working memory: visual/ } \\
\text { visuospatial }\end{array}$ & $\begin{array}{l}\text { Rey-Osterrieth Complex Figure } \\
\text { Test }^{(20,24,71,91)}\end{array}$ & Positive: flavonoid ${ }^{(20,91)}$ \\
\hline $\begin{array}{l}\text { Working memory: visual/ } \\
\text { visuospatial }\end{array}$ & $\begin{array}{l}\text { Spatial Working Memory Task } \\
\text { (CANTAB) }^{(26,80)}\end{array}$ & Positive: $\mathrm{Zn}^{(80)}$ \\
\hline $\begin{array}{l}\text { Working memory: visual/ } \\
\text { visuospatial }\end{array}$ & $\begin{array}{l}\text { Spatial Working Memory Task } \\
(\mathrm{CDR})^{(90)}\end{array}$ & Positive: flavonoid(90) \\
\hline Memory: episodic - verbal & Activity Recall $^{(79)}$ & - \\
\hline Memory: episodic - verbal & $\begin{array}{l}\text { Buschke Selective Reminding } \\
\text { Test }^{(20)}\end{array}$ & - \\
\hline Memory: episodic - verbal & $\begin{array}{l}\text { Common Objects Recall } \\
\text { Test }^{(19,21,22)}\end{array}$ & Positive: flavonoid ${ }^{(19,21,22)}$ \\
\hline Memory: episodic - verbal & $\begin{array}{l}\text { Delayed Recall of Words and } \\
\text { Pictures (CANTAB) }{ }^{(26)}\end{array}$ & - \\
\hline Memory: episodic - verbal & $\begin{array}{l}\text { Delayed Recognition Memory } \\
\text { Task }^{(83)}\end{array}$ & - \\
\hline Memory: episodic - verbal & Hong Kong List-Learning Test ${ }^{(24,89)}$ & - \\
\hline Memory: episodic - verbal & $\begin{array}{l}\text { Logical Memory Test I \& II } \\
\left(_{(\mathrm{WMS})^{(86,88,91)}}\right.\end{array}$ & - \\
\hline Memory: episodic - verbal & Memory 1 and 2 Tests (WMS) $)^{(85)}$ & - \\
\hline Memory: episodic - verbal & Memory - Probe Recall (CAT) ${ }^{(81)}$ & - \\
\hline Memory: episodic - verbal & $\begin{array}{l}\text { Memory - Recognition Memory Test } \\
(\text { CAT })^{(81)}\end{array}$ & - \\
\hline Memory: episodic - verbal & Paragraph Recall Test $(\mathrm{WMS})^{(20,72)}$ & Negative: $B$ vitamins ${ }^{(72)}$ \\
\hline Memory: episodic - verbal & $\begin{array}{l}\text { Recognition Memory Test For } \\
\text { Words }\end{array}$ & - \\
\hline Memory: episodic - verbal & $\begin{array}{l}\text { Rey Auditory-Verbal Learning } \\
\text { Test }^{(28,72,75,79,92)}\end{array}$ & $\begin{array}{l}\text { Positive: B vitamins } \text { (79) }^{(79)} \\
\text { Positive: flavonoid }{ }^{(92)}\end{array}$ \\
\hline Memory: episodic - verbal & Short Story Recall (WMS) $)^{(19,21,22)}$ & Positive: flavonoid ${ }^{(19,21)}$ \\
\hline Memory: episodic - verbal & $\begin{array}{l}\text { Verbal Memory } 1 \text { and } 2 \text { Tests } \\
\text { (WMS) }^{(85)}\end{array}$ & - \\
\hline Memory: episodic - verbal & $\begin{array}{l}\text { Verbal Paired Associates Test } \\
(\text { (WMS) })^{(91)}\end{array}$ & Positive: flavonoid ${ }^{(91)}$ \\
\hline Memory: episodic - verbal & $\begin{array}{l}\text { Word Learning Tests (Immediate } \\
\text { and Delayed Recall/Recogni- } \\
\text { tion): 10-Word Verbal Memory } \\
\text { Test/12-Word Learning Test/ } \\
\text { 15-Word Learning Test/ } \\
\text { 20-Word Free Recall Task/ } \\
\text { 30-Word Recall Test/Word } \\
\text { Learning Test/Word Recognition } \\
\text { Test (CDR) }\end{array}$ & $\begin{array}{l}\text { Positive: B vitamins }{ }^{(77)} \\
\text { Positive: multivitamins/ } \\
\text { multiminerals( }{ }^{\left({ }^{8}\right)}\end{array}$ \\
\hline ry: episodic - verbal & Word Scan Task ${ }^{(65)}$ & - \\
\hline
\end{tabular}

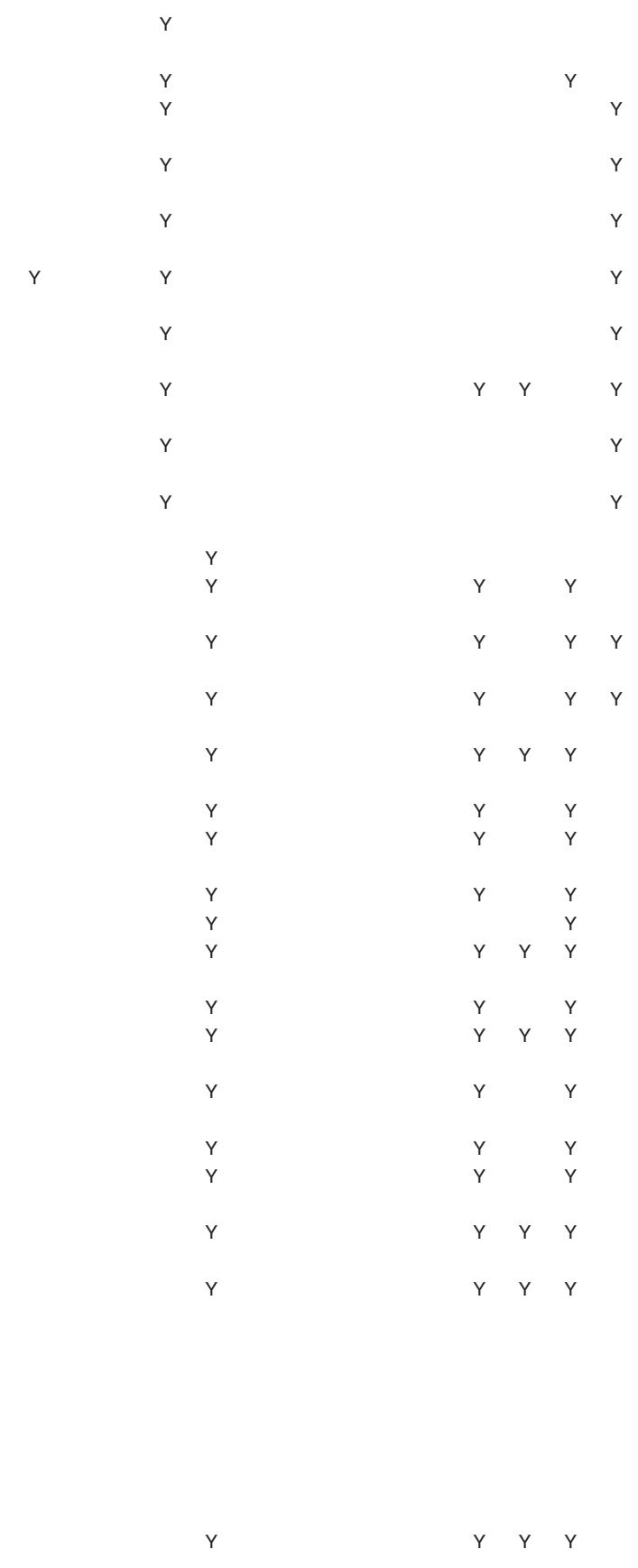




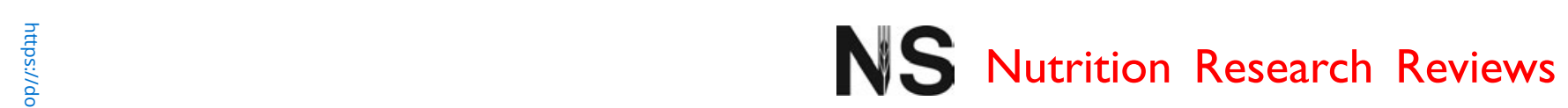

Table 2. Continued

\begin{tabular}{|c|c|c|c|c|c|c|c|c|c|c|c|c|c|c|c|c|c|c|c|c|c|c|c|c|c|c|}
\hline \multirow[b]{2}{*}{$\begin{array}{l}\text { Primary } \\
\text { neuropsychological } \\
\text { focus }\end{array}$} & \multirow[b]{2}{*}{ Task and RCT reference } & \multirow[b]{2}{*}{$\begin{array}{l}\text { Significant positive or } \\
\text { negative treatment effects }\end{array}$} & \multicolumn{7}{|c|}{ Executive function } & \multicolumn{12}{|c|}{ Memory } & \multicolumn{2}{|c|}{ Motor } & \multicolumn{3}{|c|}{ IQ } \\
\hline & & & 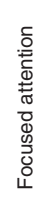 & 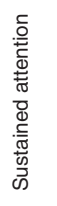 & $\begin{array}{c}c \\
\\
\end{array}$ & 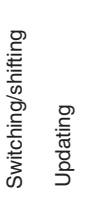 & 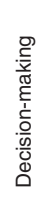 & 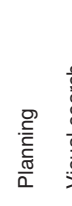 & 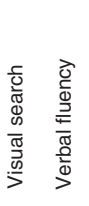 & 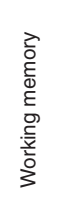 & 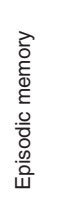 & 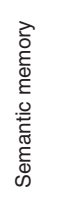 & 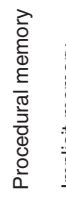 & 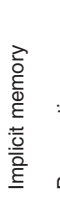 & 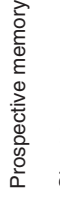 & 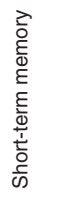 & 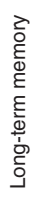 & 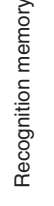 & 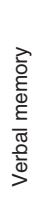 & 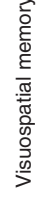 & 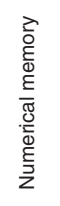 & 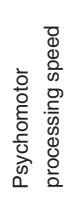 & 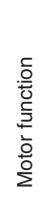 & 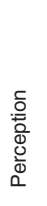 & 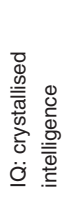 & 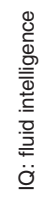 \\
\hline $\begin{array}{l}\text { Memory: episodic - } \\
\text { visual/visuospatial }\end{array}$ & $\begin{array}{l}\text { Attention - Stimulus Discrimination } \\
(\text { CAT })^{(81)}\end{array}$ & - & & & & & & & & & Y & & & & & & Y & & & Y & & & & & & \\
\hline $\begin{array}{l}\text { Memory: episodic - } \\
\text { visual/visuospatial }\end{array}$ & Benton Visual Retention Test ${ }^{(27)}$ & - & & & & & & & & & Y & & & & & & Y & & & Y & & & & & & \\
\hline $\begin{array}{l}\text { Memory: episodic - } \\
\text { visual/visuospatial }\end{array}$ & Corsi Block-Tapping (WAIS) ${ }^{(27,91)}$ & Positive: flavonoid ${ }^{(91)}$ & & & & & & & & & $\mathrm{Y}$ & & & & & & $\mathrm{Y}$ & & & Y & & & & & & \\
\hline $\begin{array}{l}\text { Memory: episodic - } \\
\text { visual/visuospatial }\end{array}$ & $\begin{array}{l}\text { Delayed Matching To Sample Test } \\
\text { (CANTAB) })^{(19,21,22)}\end{array}$ & Positive: flavonoid ${ }^{(21)}$ & & & & & & & & & Y & & & & & Y & Y & & & Y & & & & & & \\
\hline $\begin{array}{l}\text { Memory: episodic - } \\
\text { visual/visuospatial }\end{array}$ & Doors Test ${ }^{(28)}$ & - & & & & & & & & & Y & & & & & & Y & & & Y & & & & & & \\
\hline $\begin{array}{l}\text { Memory: episodic - } \\
\text { visual/visuospatial }\end{array}$ & $\begin{array}{l}\text { Pattern Recognition/Pattern } \\
\text { Recognition Memory } \\
\text { (CANTAB) })^{(26,27,80)}\end{array}$ & - & & & & & & & & & $\mathrm{Y}$ & & & & & & Y & Y & & Y & & & & & & \\
\hline $\begin{array}{l}\text { Memory: episodic - } \\
\text { visual/visuospatial }\end{array}$ & $\begin{array}{l}\text { Spatial Recognition Memory } \\
\left(^{(\text {CANTAB) }}{ }^{(26)}\right.\end{array}$ & - & & & & & & & & & Y & & & & & & Y & Y & & Y & & & & & & \\
\hline $\begin{array}{l}\text { Memory: episodic - } \\
\text { visual/visuospatial }\end{array}$ & Thurstone's Picture Memory Test ${ }^{(78)}$ & - & & & & & & & & & Y & & & & & & Y & & & Y & & & & & & \\
\hline $\begin{array}{l}\text { Memory: episodic - } \\
\text { visual/visuospatial }\end{array}$ & $\begin{array}{l}\text { Visual Memory } 1 \text { and } 2 \text { Tests } \\
\text { (WAIS) }^{(85,88)}\end{array}$ & - & & & & & & & & & Y & & & & & & Y & & & Y & & & & & & \\
\hline $\begin{array}{l}\text { Memory: episodic - } \\
\text { visual/visuospatial }\end{array}$ & Visual Reproduction Test ${ }^{(78)}$ & - & & & & & & & & & Y & & & & & & Y & & & Y & & & & & & \\
\hline Memory: semantic & Boston Naming Test ${ }^{(20,24,28,85,89)}$ & - & & & & & & & & & & $\mathrm{Y}$ & & & & & & & & & & & & & & \\
\hline Memory: semantic & Similarities Test ${ }^{(71,85,91)}$ & Positive: flavonoid ${ }^{(91)}$ & & & & & & & & & & $\mathrm{Y}$ & & & & & & & & & & & & & & \\
\hline Memory: semantic & $\begin{array}{l}\text { Spot The Word Vocabulary Test } \\
\quad\left(\text { SCOLP) }{ }^{(79)}\right.\end{array}$ & - & & & & & & & & & & Y & & & & & & & Y & & & & & & & \\
\hline Memory: semantic & Synonyms Task ${ }^{(78)}$ & Positive: $B$ vitamins ${ }^{(78)}$ & & & & & & & & & & $\mathrm{Y}$ & & & & & & & & & & & & & & \\
\hline Memory: short term & Digit Recall (WMS) ${ }^{(85)}$ & - & & & & & & & & & & & & & & $\mathrm{Y}$ & & & $\mathrm{Y}$ & & $\mathrm{Y}$ & & & & & \\
\hline Memory: short term & $\begin{array}{l}\text { Digit Span Forward } \\
\qquad \text { (WAIS) }{ }^{(24,27-29,67,71,78,84,91,92)}\end{array}$ & - & & & & & & & & & & & & & & $\mathrm{Y}$ & & & Y & & Y & & & & & \\
\hline Memory: short term & Visual Spatial Learning Test ${ }^{(20)}$ & Positive: flavonoid ${ }^{(20)}$ & & & & & & & & & $\mathrm{Y}$ & & & & & $\mathrm{Y}$ & & & & $\mathrm{Y}$ & & & & & & \\
\hline Memory: short term & $\begin{array}{l}\text { Spatial Span/Spatial Span } \\
\quad(\text { CANTAB })^{(80)}\end{array}$ & - & & & & & & & & & & & & & & Y & & & & Y & & & & & & \\
\hline Memory: short term & $\begin{array}{l}\text { Sternberg Memory Scanning } \\
\text { Task }\end{array}$ & - & & & & & & & & Y & & & & & & Y & & & & & & & & & & \\
\hline $\begin{array}{l}\text { Psychomotor: processing } \\
\text { speed }\end{array}$ & Attention - Reaction Time $(\mathrm{CAT})^{(81)}$ & Positive: $\mathrm{Fe}^{(81)}$ & & & & & & & & & & & & & & & & & & & & Y & $\mathrm{Y}$ & & & \\
\hline $\begin{array}{l}\text { Psychomotor: processing } \\
\text { speed }\end{array}$ & Boxes Test ${ }^{(79)}$ & - & & & & & & & & & & & & & & & & & & & & Y & $\mathrm{Y}$ & & & \\
\hline $\begin{array}{l}\text { Psychomotor: processing } \\
\text { speed }\end{array}$ & $\begin{array}{l}\text { Choice Reaction Time tasks: Choice } \\
\text { Reaction Time/Choice Reaction } \\
\text { Time (CDR)/Four-Choice Reac- } \\
\text { tion Time/Five-Choice Reaction } \\
\text { Time (CANTAB) }{ }^{(65,73,80,90)}\end{array}$ & Positive: multivitamins ${ }^{(65)}$ & & & & & Y & & & & & & & & & & & & & & & Y & $\mathrm{Y}$ & & & \\
\hline
\end{tabular}



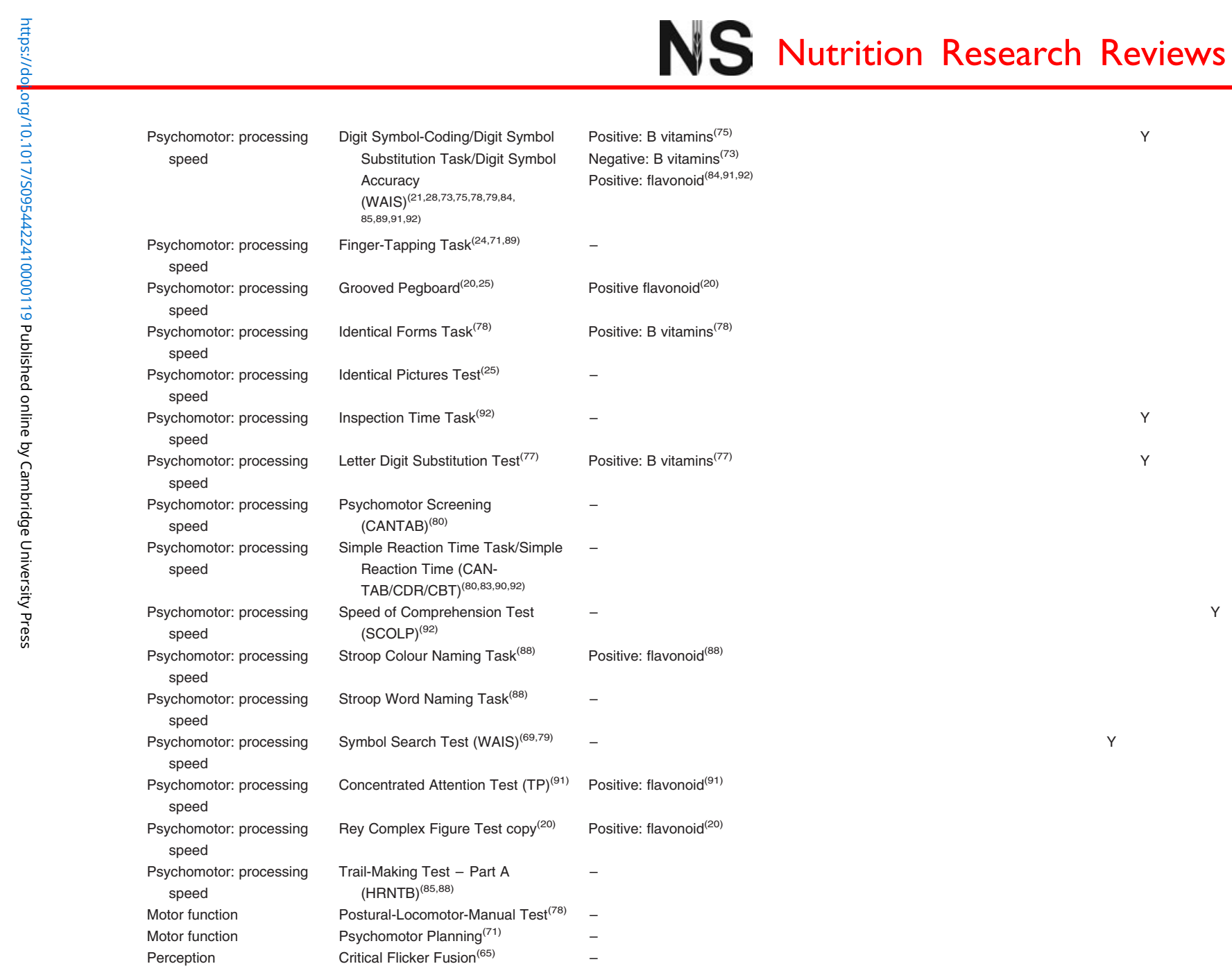

Q, intelligence quotient; Y, yes; WMS, Wechsler Memory Scale; WAIS, Wechsler Adult Intelligence Scale; CAT, Cognitive Abilities Test; Exec Fn, Executive function; IDED, Intra Dimensional/Extra Dimensional Set Shifting Task; CANTAB, Cambridge Neuropsychological Test Automated Battery; CDR, cognitive drug research; HRNTB, Halstead-Reitan Neuropsychological Test Battery; CBT, Cognometer Battery of Tests; SCOLP, Speed and Capacity of Language-Processing Test; TP, Toulouse-Pieron Test. 
The working memory span task (the Digit Span Backward test) showed significant effects in only two of eight studies, for flavonoids ${ }^{(84,92)}$, although as mentioned earlier, it is difficult to judge the quality of these results due to the lack of statistical rigour to which the data were subjected, and/or to lack of information provided.

\section{Memory}

In terms of the other memory processes under investigation, thirty-one studies investigated episodic memory, or memory for events. Episodic memory tests included both verbal and visual tasks, such as word learning, paragraph recall, picture recall, recall of common objects and delayed matching to sample. Tasks showed significant treatment effects in a quarter of the studies: five flavonoid $\mathrm{RCT}^{(19,21,22,91,92)}$, two B vitamin $\mathrm{RCT}^{(77,79)}$ and one multivitamin and mineral $\mathrm{RCT}^{(68)}$. A negative effect of treatment was found for B vitamins ${ }^{(72)}$.

As can be seen from Table 2, verbal episodic memory tasks such as the Rey Auditory Verbal Learning Test and other word learning tests showed some sensitivity to B vitamin, multivitamin and mineral, and flavonoid treatments, whereas visual or visuospatial episodic tasks were more sensitive to flavonoid treatment.

Only six of the episodic memory tests are described as such in five of the RCT under review ${ }^{(19,21,22,26,28)}$ (see Table 2). Nineteen other episodic memory tasks were defined instead as testing verbal memory. Twelve further tasks were defined as visual, spatial, or visuospatial memory tasks. In total, therefore, thirty-seven of the reviewed tasks could be described as measuring episodic memory. This includes any verbal or visuospatial memory tasks without a major working memory element, but excludes memory span measures, which are judged to measure short-term memory.

There was a wide range of tasks designed to measure verbal episodic memory, including immediate and delayed recall and/or recognition of between ten and thirty words (see 'Word-Learning Tests' in Table 2), immediate and delayed paragraph recall, and paired-associates cued-recall, all of which may impose different levels of cognitive demand, and access different types of cognitive processes and domains. Visuospatial episodic measures encompassed an equally wide range of tasks, ranging from picture recall and reproduction, for example, the Benton Visual Retention Test, to memory for faces, for example, the Wechsler Memory Scale ${ }^{(96)}$ Faces I and Faces II subscale. Immediate and delayed recall or recognition for the same task are counted here as a single memory task, for example, the Wechsler Memory Scale Logical Memory Test I and II, Memory 1 and 2 Tests, Verbal Memory 1 and 2 Tests and the Wechsler Adult Intelligence Scale ${ }^{(97)}$ Visual Memory 1 and 2 Tests.

Eight studies measured semantic memory $^{(20,24,28,71,78,79,85,89)}$, but only one vitamin B RCT showed any positive effect of treatment ${ }^{(78)}$. Again, there was a wide variation between the tasks: the Spot The Word Vocabulary Test requires participants to differentiate between words and non-words ${ }^{(79)}$, whereas the Wechsler Adult Intelligence Scale Similarities Test ${ }^{(71,85,91)}$ requires participants to describe similarities between sets of nouns.
The Boston Naming Test measures the ability to name picture objects ${ }^{(20,24,28,85,89)}$, and the Synonyms task measures the ability to select correct synonyms for given words $^{(78)}$.

Short-term memory, as measured by the digit span or recall tasks and the Sternberg Memory Scanning Task, was measured in thirteen studies. While a decline was found in a flavonoid study ${ }^{(85)}$, this effect disappeared after the authors corrected for type I error. No other effects of treatment were found for short-term memory measures.

As can be seen from Table 2, cognitive domains which have yet to be explored in micronutrient human RCT include procedural, implicit and prospective memory; it is therefore not possible to provide a more comprehensive assessment of the relationship between these dietary components and the full range of memory processes at this stage.

\section{Psychomotor/motor function}

Psychomotor processing speed was measured in twentythree studies, using a total of nineteen psychomotor tasks. The most popular type of test, used in eleven studies, was the Wechsler Adult Intelligence Scale Digit Symbol Substitution or Digit Symbol Coding Test, designed as a measure of psychomotor processing speed ${ }^{(62)}$ but often used as a measure of working memory. These tasks were shown to be sensitive to flavonoid ${ }^{(84,88,91,92)}$ and vitamin $\mathrm{B}^{(73,75)}$ treatments. Three other psychomotor processing speed tasks were also sensitive to vitamin B treatment: the Boxes Test ${ }^{(79)}$, Identical Forms Task $^{(78)}$ and Letter Digit Substitution Test ${ }^{(77)}$. The next most popular paradigm was choice reaction time (CRT), a complex form of the simple reaction time (SRT) paradigm used in four RCT. The CRT task also has a decision-making component and was sensitive to a multivitamin intervention ${ }^{(65)}$, unlike the SRT which was non-significant across four studies ${ }^{(80,83,90,92)}$.

\section{Multiple cognitive measures}

Within a domain, the number of measures used varied widely from study to study. Whereas the majority selected one or two measures to represent a single cognitive domain, Santos et al. ${ }^{(91)}$ used twenty-two measures overall, including ten executive function tasks, six memory tests and four IQ performance measures. Bryan et al. ${ }^{(79)}$ used eight measures of executive function and working memory, and five other memory tasks. Gleason et al. ${ }^{(20)}$ used six executive function tasks and four memory measures and Howes et al. ${ }^{(85)}$ used four executive function tasks and six memory measures.

One advantage of the multiple measures approach is that it provides an opportunity for researchers to compute a composite measure of a particular domain, thus controlling in part for the idiosyncrasies of individual tasks and the potential for type I error. Murray-Kolb \& Beard $^{(81)}$ and Ryan et al. ${ }^{(90)}$ used factor analysis to group 'families' of tasks to reduce the number of cognitive variables. Neither of these studies found significant results using these composite scores. Notwithstanding this, the approach has some promise, although caution is needed when deriving 
composite measures, as cognitive tasks are likely to be correlated and therefore some statistical techniques may not be warranted.

With an increasing number of cognitive tasks there comes an increased danger of type I error during analysis. This issue was dealt with in two cases ${ }^{(22,27)}$ by using multiple ANOVA, which reduces overall error.

\section{Global cognitive measures}

Five studies targeted general rather than specific cognition, using only general or 'global' measures of cognitive functions such as the MMSE, the ADAS-Cog and Hasegawa's Dementia Rating Scale ${ }^{(23,66,74,76,82)}$. Two RCT relied solely on the MMSE or the ADAS-Cog for measurement of cognitive function: one of these studied the effects of flavonoids on Alzheimer's disease patients, showing a significant positive effect of flavonoid treatment over 12 months ${ }^{(23)}$. The other found no effect of vitamin $\mathrm{B}$ treatment in older adults with a MMSE score ranging from 6 to 28 out of a possible $30^{(74)}$. Overall, the MMSE was used as a performance measure in fourteen $\operatorname{RCT}^{(24,28,65,66,68,70-74,76,82,86,89)}$ but only showed a significant effect of treatment in one ${ }^{(89)}$, although this may have been due to the inclusion of cognitively impaired controls with a baseline MMSE score of less than 24 out of 30 . The ADAS-Cog was used in four studies ${ }^{(23,66,76,82)}$, and showed significant effects of treatment for patients with a baseline MMSE score of less than 24 in one 12-month flavonoid Alzheimer's disease intervention ${ }^{(23)}$, with the placebo group showing a significantly greater decline on the ADAS-Cog than the treatment group.

\section{Cognitive screening and baseline population measures}

Twelve studies (31\%) included participants with MMSE scores of less than 24 out of 30, which is suggestive of cognitive impairment or dementia $^{(23,24,29,66,68,70,71,74,76,82,83,91)}$. Eighteen studies $(46 \%)$ did not screen for cognitive impairment; of these, thirteen included participants over the age of 50 years $^{(19,22,25,27,28,65,69,78,79,84,86,89,90)}$ and may also, therefore, have unwittingly included cognitively impaired participants. Three studies reported baseline statistical differences in cognitive performance ${ }^{(68,69,91)}$. Eleven studies did not report statistical baseline comparisons for any cognitive performance measures, making it difficult to assess the efficacy of their randomisation pro$\operatorname{cess}^{(19,20,26,27,81-84,88,89,92)}$, and Bryan et al. ${ }^{(79)}$ only provided this information for some of their cognitive tasks. Casini et al. ${ }^{(84)}$ collected cognitive data at the end of each treatment arm in their cross-over design, thereby providing no real baseline cognitive data.

In general, IQ tests were used to provide measures of possible baseline differences between treatment groups, although Santos et al. ${ }^{(91)}$ used four crystallised intelligence IQ tasks as performance measures and, unusually, found significant differences after 8 months of treatment with G. biloba. Examination of means shows that the treatment group had generally lower baseline IQ scores but similar endpoint scores on these measures, suggesting a measurement correction rather than a treatment effect.

\section{Reasons given for null results}

Fourteen RCT were considered by their authors to be underpowered with too-small sample sizes. Sixteen suggested that the duration of the treatment was too short, in interventions that ranged from 4 weeks $^{(73,74)}$ to 2 years $^{(72)}$. Two suggested that the doses used were inadequate $^{(24,72)}$. Authors of six RCT warned that the results may not be generalisable across other populations ${ }^{(25,72,76,77,80,92)}$; for instance, as the authors pointed out, vitamin B trials may have different results when carried out in countries where fortification with folic acid is mandatory ${ }^{(76,77)}$. With a few exceptions ${ }^{(65,87,88)}$, researchers have not focused on the sensitivity of the cognitive measures when explaining null results.

\section{Discussion}

Less than half the studies reviewed showed any positive treatment effects of target nutrients. In terms of looking at efficacy for individual cognitive tasks, this equates to only thirty-eight out of 121 (31\%) displaying some sensitivity to chronic supplementation. This is consistent with findings from other reviews of nutrition and prevention of cognitive decline, such as Hoyland et al. ${ }^{(98)}$, Jia et al. ${ }^{(17)}$ and Macready et al. (18). Hoyland et al. ${ }^{(98)}$, for instance, found that in macronutrient intervention studies, the most significant performance differences occurred with the most demanding tasks and with delayed memory performance. It is possible that the tasks showing the greatest sensitivity in micronutrient and flavonoid interventions may also impose the greatest cognitive demands and/or memory performance stimulus-response delays; this is certainly worth considering for systematic investigation in the future, but it has not yet been explored in the field of micronutrient or flavonoid human chronic RCT research.

The quality-grading exercise revealed only six studies that could be considered as examples of best practice: three vitamin B studies $^{(70,71,77)}$, two flavonoid studies ${ }^{(24,28)}$ and a $\mathrm{Zn} \mathrm{RCT}^{(80)}$. The remainder failed to meet one or more of the criteria set out in the Methods section for category A, including: inappropriate comparator, outcome measure, statistical method and/or reporting; insufficient power; reporting errors; and unclear description of the population, setting or reporting of drop-outs. Ideally, there should be less than $20 \%$ drop-out and RCT should be double-blinded where possible. While findings were mixed, and interpretation of the findings is made more challenging by methodological shortcomings in individual studies, some cognitive domains did show more sensitivity to nutritional supplementation than others. Specifically, there were reports of positive treatment effects on tasks with a spatial memory component with $\mathrm{Zn}$ and flavonoids in two RCT, one of which was graded category $\mathrm{A}^{(80)}$. Vitamin B and Fe showed benefits for psychomotor processing speed, with the vitamin $\mathrm{B}$ study also classified as a category $\mathrm{A} \mathrm{RCT}^{(77)}$, although this was not supported by findings from the two other category A vitamin B studies ${ }^{(70,71)}$. Flavonoids also showed 
positive effects for executive function, in particular frontal function (Intra Dimensional/Extra Dimensional Set Shifting Task Rule Learning and Reversal Task), inhibition (Stroop Colour-Word Task), planning (Stockings of Cambridge Task), sustained attention (Paced Auditory Serial Addition Test), and category fluency tasks, although some of these findings are from category $\mathrm{C}$ studies and should therefore be interpreted with a degree of caution. In addition, $\mathrm{Zn}$ generated an improvement on a visual search task (Match to Sample $)^{(80)}$. Finally, there were also some nutrient effects on various episodic memory tasks, in particular the Common Objects Recall Test, the Rey Auditory-Verbal Learning Test, a short story recall task and various word learning tests, and the Delayed Matching To Sample Test, a measure of visuospatial episodic memory ${ }^{(19,21,22,68,77,79,92)}$. While some of these findings are from category $\mathrm{C}$ studies, the majority are from higher-graded studies $(21,22,77,79)$.

Interestingly, some of the domain-specific nutrient effects shown here in human RCT studies overlap quite closely with findings reported in animal studies. For example, in rat studies, positive effects of flavonoid intake have been shown on spatial memory tasks ${ }^{(10,99)}$ and psychomotor performance $^{(100)}$. In animal models, these tasks are thought to involve brain regions such as the hippocampus, which is associated with place learning ${ }^{(101)}$, the basal ganglia and striatum which are linked to cue and response learning ${ }^{(102)}$, and the prefrontal cortex, which is central to rule acquisition in procedural learning ${ }^{(103)}$. In rats, these same regions have been shown to be responsive to treatment by flavonoids, for instance via increased striatal muscarinic receptor sensitivity $^{(10,16,99,104,105)}$. In terms of mechanisms, there is some suggestion that flavonoids may improve cell signalling which in turn may enhance neuroprotection through their antioxidant and anti-inflammatory properties, and may also promote neurogenesis. According to neuroimaging studies, similar brain regions are involved in human tasks. For example, areas including the hippocampal formation have been implicated in spatial memory ${ }^{(48)}$, whereas psychomotor processing speed may include a striatal component ${ }^{(50)}$. Whether or not the mechanisms of action are the same in both animals and humans remains to be seen.

Despite strong evidence from animal studies of a positive relationship impact of certain nutrients on cognitive function, these important findings do not appear to be driving RCT research in human studies. In human flavonoid research, for instance, there appears to be little explicit reference to animal work in the search for 'primary dependent measures' to use in human flavonoid intervention studies. As an example, spatial memory was explored in only $65 \%$ of flavonoid studies, despite evidence from animal work that spatial processing may be the cognitive domain which is most sensitive to flavonoid intervention. While only $20 \%$ of those studies that included a task with a spatial memory component showed positive results, it is noteworthy that those specifically designed to measure 'spatial working memory' did show some nutrient-driven improvements (see Table 2, under 'Working memory: visual/visuospatial'). This may have been due to the level of cognitive demand, or to the number and complexity of cognitive functions or processes required to perform the task. One key difference between animal and human studies though is that spatial tasks used in animal studies involve the rodent subject moving within a three-dimensional space with ever-changing environmental cues. In human studies, spatial tasks tend to be presented in a two-dimensional environment, arguably involving very different kinds of cognitive functioning to those employed in animal studies. While a number of flavonoid studies used memory tasks with a visual or visuospatial element, few have attempted to measure spatial working memory in a similar way to that in which it is assessed in animal paradigms; this is particularly true of cognitive processes and functions that are normally employed in three-dimensional maze task environments such as navigation and orientation. Better analogues of animal tasks or more similarly demanding tasks should be developed, perhaps using new technologies such as virtual reality (see, for example, Astur et al. ${ }^{(106)}$ ).

While some preliminary conclusions can be drawn from the available data, the ability to do so is hindered by an inconsistent approach to cognitive testing in nutrition supplementation studies, particularly in terms of the cognitive domains measured and tasks employed. Some researchers targeted specific domains such as memory or executive function, whereas others measured multiple domains. Both single and multiple domain studies included different numbers of measures within each cognitive domain, varying from single measures per domain to multiple measures, and researchers treating cognitively impaired populations were limited to using general measures of cognitive function for ethical and/or practical reasons. There is therefore little correspondence between the approaches taken in the measurement of cognitive function within or across nutrients, making it difficult to build a comprehensive picture of the relationship between nutrition and cognitive function.

It is also clear from looking at Table 2 that while some cognitive domains have been covered fairly extensively, for instance, episodic memory, others, such as semantic memory, have not. There are also a number of key gaps in the domains measured where no RCT research has been carried out at all, namely implicit, procedural, and prospective memory. Collectively, procedural memory and implicit memory are involved in skill learning ${ }^{(107,108)}$. They are therefore important to study from a practical perspective, particularly for every-day cognitive functioning in older human adults, and there is strong evidence that the striatum, previously shown to be sensitive to nutrient supplementation in animal models ${ }^{(10)}$, is involved in procedural memory ${ }^{(109)}$. Furthermore, implicit compared with explicit memory tasks are thought to make minimal demands on hippocampal-based brain regions ${ }^{(110)}$; as many animal studies have focused on the effects of nutrients on cognition using tasks with a significant hippocampal component, the inclusion of implicit memory tasks would provide a good empirical test of the degree to which benefits observed for particular nutrients are brain-region specific. Prospective memory declines in older adulthood, especially when the task makes demands on the provision and allocation of attentional/executive resources ${ }^{(111)}$, as it recruits areas of the prefrontal cortex and parietal cortex $^{(112)}$, and is also therefore worthy of exploration. Overall, distinctions between implicit $v$. explicit memory, 
procedural $v$. declarative memory, and prospective $v$. retrospective memory represent major taxonomic divisions in human memory and cannot simply be ignored.

Another source of inconsistency comes from the wide range of measures used across studies, in particular those exploring the same nutrient, making comparability between studies and within domains difficult. Not only has a large number of tasks been used within a single domain, the variability between those tasks is also a concern. For instance, the verbal memory tasks varied enormously from each other, from recall (immediate and delayed) and recognition of word lists, paragraphs and stories to pairedassociates recall; the word lists in the memory tasks ranged from ten to thirty words; semantic memory tasks measured (1) naming of objects, (2) differentiation of words and nonwords, (3) similarities between nouns and (4) synonyms for given words; and two spatial working memory tasks differed in the type of stimuli shown and the level of complexity of the task. So while all these tasks may be measuring cognitive function within the same primary domain, they may also impose different levels of cognitive demand, and involve different processes or even different secondary cognitive domains. One sensible approach may therefore be to include at least two measures of a cognitive domain of interest where possible.

One of the more concerning findings in the present review is that researchers continue to use cognitive tests that seemingly display little sensitivity to nutritional supplementation. The MMSE, recommended for use as a screening tool for cognitive impairment, was used as a performance measure in $38 \%$ of the studies but failed to show significance in all but one, suggesting either that the nutrient in question has no effect on general cognitive function, or that the MMSE is not sensitive enough to measure short-term micronutrient- or flavonoid-driven cognitive change. While Woo et al. ${ }^{(89)}$ did find significant positive effects of flavonoid treatment on postmenopausal women on three cognitive tasks including the MMSE (which may have been due to differences in baseline scores), Cockle et al. ${ }^{(65)}$ found no change using the MMSE but a positive effect of treatment using a choice reaction time task, which suggests that the issue here may be one of test sensitivity rather than lack of treatment efficacy. The MMSE may simply not be sensitive enough to capture the subtle cognitive changes associated with dietary treatments, particularly with younger and/or cognitively healthy populations who are likely to perform at ceiling. Over half the reviewed studies which used the MMSE as a performance measure had normal populations without dementia or mild cognitive impairment, which may also have contributed to the large number of null findings. Similarly, the Trail-Making Task was only significant in two out of thirteen studies, showing a negative effect of treatment in both cases. The Boston Naming Test was used as a measure of semantic memory in six studies, including two category $\mathrm{A} \mathrm{RCT}^{(24,28)}$, but was not significant in any. Of course, such consistently negative results may suggest that nutritional supplementation has no effect, or even a detrimental effect, on the specific cognitive function(s) measured by these tasks. As positive treatment effects have been observed with other, similar tasks though, it is more likely a question of task sensitivity and/or intensity of task demand. The possibility of type I error and lack of power in relation to effect size should also be considered. Therefore, while replication of cognitive methodologies is important, it would be good practice to move away from measures that repeatedly show null results and to identify tasks that are more consistently sensitive to treatment in the target populations.

There is also considerable confusion in the literature concerning the terms used to describe the same task. As one example, the Trail-Making Task Parts A and B have been referred to by different researchers as measuring visuomotor tracking and attention ${ }^{(86)}$; speed for attention, sequencing, mental flexibility, visual search and motor function ${ }^{(24)}$; information processing and prefrontal lobe function ${ }^{(85)}$; sequencing and shifting perceptual sets, concentration/ vigilance and visuomotor scanning/tracking speed ${ }^{(88)}$. While all these terms may have some validity, this inconsistency may lead to confusion for future researchers, and may hinder systematic comparison and interpretation of the specific tasks across studies. There were also discrepancies between what tasks actually measured. For instance, the Similarities Test was categorised by Eussen et al. ${ }^{(71)}$ as executive function, by Howes et al. ${ }^{(85)}$ as verbal reasoning ${ }^{(85)}$ and as an aspect of general intelligence by Santos et al. ${ }^{(91)}$. In a number of cases, the tasks have been listed with no description and the reader is simply referred to the manual. This is especially true of Wechsler Adult Intelligence Scale and Wechsler Memory Scale tasks, which have undergone a number of updates over the years, and researchers may not have access to particular editions. Without access to the manuals it is often difficult to assess the suitability and sensitivity of tasks for future research.

The focus of much of the research reviewed here currently centres on identifying the correct dose and duration of treatment to bring about improvements in cognitive function, and adequately powering the study to enable significant differences to be shown. Authors of approximately a third of the studies reviewed suggested that their sample sizes were too small to provide adequate statistical power, or that their significant findings were a result of type I error. Those who had carried out power calculations often had to base their calculations on previous research in another area, or on projected changes in biomarkers rather than increments in cognitive change, due to the lack of availability of more appropriate data. Some authors warned that their results may not be generalisable to other populations. While there clearly are inconsistencies of approach in terms of intervention dose and duration, and issues about statistical power, sample size and type I error, it is our view that definitive conclusions regarding the efficacy of the dose or duration of a particular nutrient cannot be made until the sensitivity of the cognitive measures used has been established for the type of nutrient under investigation. This may be achievable through, for instance, the systematic manipulation of cognitive demand in an acute design, as seen in the glucose RCT study literature, where glucose was shown to be of greater benefit on more cognitively demanding tasks ${ }^{(98,113)}$.

Further consideration of individual differences such as age needs to be given when selecting cognitive tests. 
For example, as can be seen from Table 1, less than a quarter of the studies were carried out on younger adults. As it is possible that the optimal window of opportunity for slowing or reversal of age-related declines by dietary means may occur much earlier in the life cycle, younger populations should be given greater consideration. Clearly tests such as the MMSE are unlikely to be sensitive enough for younger, healthier populations, but tasks that are more challenging and sensitive for younger participants will also inform about older cognitively intact and/or at-risk populations, allow better comparability between young and older age groups, and enable a greater understanding of diet-related cognitive evolution throughout the lifespan. Screening is important, as there is a danger of including participants with mild cognitive impairment within a healthy study sample, which makes interpretation difficult, as results may not be transferable to cognitively healthy populations. Finally, reporting of statistical differences in cognitive scores at baseline is essential, as this enables an informed assessment of parity between treatment groups based on initial cognitive performance.

\section{Conclusion and recommendations}

Research on the relationship between nutrition and cognitive function clearly has a long way to go, and an increasingly complex picture is emerging. Findings from epidemiological, longitudinal, observational and animal studies suggest that certain nutrients may offer great potential in the treatment of age-related cognitive disorders, and excitingly, certain nutrients may have specific roles to play in improving specific cognitive functions. However, this potential is currently hampered by methodological difficulties, and by the unsystematic approach being adopted across studies, reducing their comparability and making interpretation of findings difficult. As well as interpretative difficulties, such a 'scattergun' approach limits the ability to make reliable comparisons across studies. The above findings demonstrate the necessity for more standardised, sensitive, and theory-derived sets of cognitive tasks in future clinical and dietary intervention studies.

The present review offers a number of implications for future chronic dietary studies. Firstly, there is a clear need to pay closer attention to animal studies and to previous human work when identifying appropriate cognitive tests, both within and across nutrients. Where reliable and nutritionally sensitive cognitive tasks are identified, researchers should endeavour to incorporate them into subsequent test batteries so that patterns relating to issues such as dose-response effects and nutrient type sensitivity can more easily emerge. Secondly, tasks need to be appropriate to the target population, be sufficiently sensitive to the nutrient under investigation, be sufficiently demanding to discriminate between good and poor performers, and capable of avoiding ceiling (and floor) effects. Thirdly, greater care should be taken to avoid statistical artifacts likely to bring about null findings, such as lack of power, and type I errors. Finally, including more than a single task within a domain (for example, two executive function tasks) would greatly help to determine whether a null effect for a particular nutrient is a real finding or reflects a lack of task sensitivity to the nutrient in question. Adopting these simple guidelines will bring much needed clarity and methodological rigour and, in the longer term, permit researchers to make much clearer policy recommendations for dietary intake in the general public.

\section{Acknowledgements}

J. P. E. S., L. T. B. and C. M. W. are sponsored by the Biotechnology and Biological Sciences Research Council (BB/F008953/1). J. P. E. S. is also funded by BBSRC grants (BB/E023185/1; BB/G005702/1; BB/C518222/1; $\mathrm{BB} / \mathrm{E} 023185 / 1)$ and the Medical Research Council (G0400278/NI02). J. P. E. S. and O. B. K. are funded by the Food Standards Agency (FSA; grant N02039). This research received no specific grant from any funding agency in the public, commercial or not-for-profit sectors.

A. L. M. and L. T. B. were responsible for paper conceptualisation and manuscript preparation; O. B. K., J. A. E., C. M. W. and J. P. E. S. contributed to paper conceptualisation and manuscript editing.

The authors are not aware of any conflicts of interest relating to this paper.

\section{References}

1. World Health Organization (2007) Medium-term strategic plan 2008-2013 and proposed programme budget 2008-2009. Geneva: WHO.

2. Knapp M, Prince M, Albanese E, et al. (2007) Dementia UK - A Report into the Prevalence and Cost of Dementia. Prepared by the Personal Social Services Research Unit (PCSSRU) at the London School of Economics and Institute of Psychiatry at King's College London for the Alzheimer's Society. London: Alzheimer's Society.

3. Wimo A, Winblad B \& Jönsson L (2007) An estimate of the total worldwide societal costs of dementia in 2005. Alzheimers Dement 3, 81-91.

4. Morris MC, Evans DA, Bienias JL, et al. (2002) Vitamin E and cognitive decline in older persons. Arch Neurol 59, $1125-1132$.

5. Perkins AJ, Hendrie HC, Callahan CM, et al. (1999) Association of antioxidants with memory in a multiethnic elderly sample using the Third National Health and Nutrition Examination Survey. Am J Epidemiol 150, 37-44.

6. de Lau LML, Refsum H, Smith AD, et al. (2007) Plasma folate concentration and cognitive performance: Rotterdam scan study. Am J Clin Nutr 86, 728-734.

7. Feng L, Ng TP, Chuah L, et al. (2006) Homocysteine, folate, and vitamin B-12 and cognitive performance in older Chinese adults: findings from the Singapore Longitudinal Ageing Study. Am J Clin Nutr 84, 1506-1512.

8. Letenneur L, Proust-Lima C, Le Gouge A, et al. (2007) Flavonoid intake and cognitive decline over a 10-year period. Am J Epidemiol 165, 1364-1371.

9. Galli RL, Shukitt-Hale B, Youdim KA, et al. (2002) Fruit polyphenolics and brain aging - nutritional interventions targeting age-related neuronal and behavioral deficits. Ann N Y Acad Sci 959, 128-132.

10. Joseph JA, Shukitt-Hale B, Denisova NA, et al. (1999) Reversals of age-related declines in neuronal signal transduction, cognitive, and motor behavioral deficits with blueberry, spinach, or strawberry dietary supplementation. J Neurosci 19, 8114-8121. 
11. Joseph JA, Shukitt-Hale B, Denisova NA, et al. (1998) Long-term dietary strawberry, spinach, or vitamin E supplementation retards the onset of age-related neuronal signal-transduction and cognitive behavioral deficits. J Neurosci 18, 8047-8055.

12. Barros D, Amaral OB, Izquierdo I, et al. (2006) Behavioral and genoprotective effects of Vaccinium berries intake in mice. Pharmacol Biochem Behav 84, 229-234.

13. Cho J, Kang JS, Long PH, et al. (2003) Antioxidant and memory enhancing effects of purple sweet potato anthocyanin and Cordyceps mushroom extract. Arch Pharm Res 26, 821-825.

14. Lau FC, Shukitt-Hale B \& Joseph JA (2005) The beneficial effects of fruit polyphenols on brain aging. Neurobiol Aging 26, S128-S132.

15. Ramirez MR, Izquierdo I, Raseira MDB, et al. (2005) Effect of lyophilised Vaccinium berries on memory, anxiety and locomotion in adult rats. Pharmacol Res 52, 457-462.

16. Shukitt-Hale B, Carey A, Simon L, et al. (2006) Effects of Concord grape juice on cognitive and motor deficits in aging. Nutrition 22, 295-302.

17. Jia X, McNeill G \& Avenell A (2008) Does taking vitamin, mineral and fatty acid supplements prevent cognitive decline? A systematic review of randomized controlled trials. J Hum Nutr Diet 21, 317.

18. Macready AL, Kennedy OB, Ellis JA, et al. (2009) Flavonoids and cognitive function: a review of human randomized controlled trial studies and recommendations for future studies. Genes Nutr 4, 227-242.

19. File SE, Hartley DE, Elsabagh S, et al. (2005) Cognitive improvement after 6 weeks of soy supplements in postmenopausal women is limited to frontal lobe function. Menopause 12, 193-201.

20. Gleason CE, Carlsson CM, Barnet JH, et al. (2009) A preliminary study of the safety, feasibility and cognitive efficacy of soy isoflavone supplements in older men and women. Age Ageing 38, 86-93.

21. File SE, Jarrett N, Fluck E, et al. (2001) Eating soya improves human memory. Psychopharmacologia 157, 430-436.

22. Duffy R, Wiseman H \& File SE (2003) Improved cognitive function in postmenopausal women after 12 weeks of consumption of a soya extract containing isoflavones. Pharmacol Biochem Behav 75, 721-729.

23. Le Bars PL, Velasco FM, Ferguson JM, et al. (2002) Influence of the severity of cognitive impairment on the effect of the Ginkgo biloba extract EGb $761^{\circledR}$ in Alzheimer's disease. Neuropsychobiology 45, 19-26.

24. Ho SC, Chan ASY, Ho YP, et al. (2007) Effects of soy isoflavone supplementation on cognitive function in Chinese postmenopausal women: a double-blind, randomized, controlled trial. Menopause 14, 489-499.

25. Basaria S, Wisniewski A, Dupree K, et al. (2009) Effect of high-dose isoflavones on cognition, quality of life, androgens, and lipoprotein in post-menopausal women. $J$ Endocrinol Invest 32, 150-155.

26. Elsabagh S, Hartley DE, Ali O, et al. (2005) Differential cognitive effects of Ginkgo biloba after acute and chronic treatment in healthy young volunteers. Psychopharmacology 179, 437-446.

27. Fournier LR, Ryan BTA, Robison LM, et al. (2007) The effects of soy milk and isoflavone supplements on cognitive performance in healthy, postmenopausal women. $J$ Nutr Health Aging 11, 155-164.

28. Kreijkamp-Kaspers S, Kok L, Grobbee DE, et al. (2004) Effect of soy protein containing isoflavones on cognitive function, bone mineral density, and plasma lipids in postmenopausal women - a randomized controlled trial. JAMA 292, 65-74.

29. van Dongen MC, van Rossum E, Kessels AG, et al. (2000) The efficacy of ginkgo for elderly people with dementia and age-associated memory impairment: new results of a randomized clinical trial. J Am Geriatr Soc 48, 1183-1194.

30. Salthouse TA (1990) Working memory as a processing resource in cognitive aging. Dev Rev 10, 101-124.

31. Waters GS \& Caplan D (2003) The reliability and stability of verbal working memory measures. Behav Res Methods Instrum Comput 35, 550-564.

32. Burgess PW (1997) Theory and methodology in executive function research. In Methodology of Frontal and Executive Function, pp. 81-116 [P Rabbitt, editor]. Hove, UK: Psychology Press.

33. Rugg MD \& Henson RNA (2002) Episodic memory retrieval: an (event-related) functional neuroimaging perspective. In The Cognitive Neuroscience of Memory Encoding and Retrieval, pp. 3-37 [AE Parker, EL Wilding and T Bussey, editors]. Hove, UK: Psychology Press.

34. Miyake A, Friedman NP, Emerson MJ, et al. (2000) The unity and diversity of executive functions and their contributions to complex 'frontal lobe' tasks: a latent variable analysis. Cognit Psychol 41, 49-100.

35. Rabbitt P (1997) Methodology of Frontal and Executive Function. Hove, UK: Psychology Press.

36. Manly T \& Robertson IH (1997) Sustained attention and the frontal lobes. In Methodology of Frontal and Executive Function, pp. 135-154 [P Rabbitt, editor]. Hove, UK: Psychology Press.

37. Phillips LH (1997) Do 'frontal tests' measure executive function? Issues of assessment and evidence from fluency tests. In Methodology of Frontal and Executive Function, pp. 191-214 [P Rabbitt, editor]. Hove, UK: Psychology Press.

38. Robbins TW (2007) Shifting and stopping: fronto-striatal substrates, neurochemical modulation and clinical implications. Philos Trans R Soc Lond B Biol Sci 362, 917-932.

39. Costafreda SG, Fu CH, Lee L, et al. (2006) A systematic review and quantitative appraisal of fMRI studies of verbal fluency: role of the left inferior frontal gyrus. Hum Brain Mapp 27, 799-810.

40. D'Esposito M (2007) From cognitive to neural models of working memory. Philos Trans R Soc Lond B Biol Sci 362, $761-772$

41. Curtis CE, Rao VY \& D'Esposito M (2004) Maintenance of spatial and motor codes during oculomotor delayed response tasks. J Neurosci 24, 3944-3952.

42. Henson RNA, Burgess N \& Frith CD (2000) Recoding, storage, rehearsal and grouping in verbal short-term memory: an fMRI study. Neuropsychologia 38, 426-440.

43. Ranganath C, Johnson MK \& D'Esposito M (2003) Prefrontal activity associated with working memory and episodic long-term memory. Neuropsychologia 41, 378-389.

44. Voss JL \& Paller KA (2008) Brain substrates of implicit and explicit memory: the importance of concurrently acquired neural signals of both memory types. Neuropsychologia 46, 3021-3029.

45. Wagner AD, Shannon BJ, Kahn I, et al. (2005) Parietal lobe contributions to episodic memory retrieval. Trends Cogn Sci 9, 445-453.

46. Voss JL, Reber PJ, Mesulam MM, et al. (2008) Familiarity and conceptual priming engage distinct cortical networks. Cerebral Cortex 18, 1712-1719.

47. Schott BH, Henson RN, Richardson-Klavehn A, et al. (2005) Redefining implicit and explicit memory: the 
functional neuroanatomy of priming, remembering, and control of retrieval. Proc Nat Acad Sci U S A 102, $1257-1262$.

48. Spiers HJ \& Maguire EA (2007) The neuroscience of remote spatial memory: a tale of two cities. Neuroscience 149, 7-27.

49. Simons JS, Schölvinck ML, Gilbert SJ, et al. (2006) Differential components of prospective memory?: Evidence from fMRI. Neuropsychologia 44, 1388-1397.

50. Mink JW (1996) The basal ganglia: focused selection and inhibition of competing motor programs. Prog Neurobiol 50, 381-425.

51. Shadmehr R \& Holcomb HH (1997) Neural correlates of motor memory consolidation. Science 277, 821-825.

52. Attebo K, Mitchell P \& Smith W (1996) Visual acuity and the causes of visual loss in Australia. The Blue Mountains Eye Study. Ophthalmology 103, 357-364.

53. Ivers R, Cumming RG, Mitchell P, et al. (1998) Visual impairment and falls in older adults: The Blue Mountains Eye Study. J Am Geriatr Soc 46, 58-64.

54. Ganis G, Thompson WL \& Kosslyn SM (2004) Brain areas underlying visual mental imagery and visual perception: an fMRI study. Cogn Brain Res 20, 226-241.

55. Cattell RB (1963) Theory of fluid and crystallized intelligence: a critical experiment. J Educ Psychol 54, $1-22$.

56. Duncan J, Seitz RJ, Kolodny J, et al. (2000) A neural basis for general intelligence. Science 289, 457-460.

57. Gray JR \& Thompson PM (2004) Neurobiology of intelligence: science and ethics. Nature Rev Neurosci 5, 471-482.

58. Folstein MF, Folstein SE \& McHugh PR (1975) MiniMental State - practical method for grading cognitive state of patients for clinician. J Psychiatr Res 12, 189-198.

59. Rosen WG, Mohs RC \& Davis KL (1984) A new ratingscale for Alzheimer's Disease. Am J Psychiatry 141, 1356-1364.

60. Petersen RC, Smith GE, Waring SC, et al. (1999) Mild cognitive impairment: clinical characterization and outcome. Arch Neurol 56, 303-308.

61. Roselli F, Tartaglione B, Federico F, et al. (2009) Rate of MMSE score change in Alzheimer's disease: influence of education and vascular risk factors. Clin Neur Neurosurg 111, 327-330.

62. Lezak MD, Howieson DB, Loring DW, et al. (2004) Neuropsychological Assessment. New York, NY: Oxford University Press.

63. Balk E, Chung M, Raman G, et al. (2006) B Vitamins and Berries and Age-Related Neurodegenerative Disorders. Evidence Report/Technology Assessment no. 134. Rockville, MD: Agency for Healthcare Research and Quality.

64. Levey AS, Coresh J, Balk E, et al. (2003) National Kidney Foundation practice guidelines for chronic kidney disease: evaluation, classification, and stratification. Ann Intern Med 139, 137-147.

65. Cockle SM, Haller J, Kimber S, et al. (2000) The influence of multivitamins on cognitive function and mood in the elderly. Aging Mental Health 4, 339-353.

66. Clarke R, Harrison G, Richards S, et al. (2003) Effect of vitamins and aspirin on markers of platelet activation, oxidative stress and homocysteine in people at high risk of dementia. $J$ Int Med 254, 67-75.

67. McNeill G, Avenell A, Campbell MK, et al. (2007) Effect of multivitamin and multimineral supplementation on cognitive function in men and women aged 65 years and over: a randomised controlled trial. Nutr J 6, 10.
68. Wouters-Wesseling W, Wagenaar LW, Rozendaal M, et al. (2005) Effect of an enriched drink on cognitive function in frail elderly persons. J Gerontol A Biol Sci Med Sci 60, 265-270.

69. Wolters M, Hickstein M, Flintermann A, et al. (2005) Cognitive performance in relation to vitamin status in healthy elderly German women - the effect of 6-month multivitamin supplementation. Prev Med 41, 253-259.

70. Hvas AM, Juul S, Lauritzen L, et al. (2004) No effect of vitamin B-12 treatment on cognitive function and depression: a randomized placebo controlled study. $J$ Affect Disord 81, 269-273.

71. Eussen SJ, de Groot LC, Joosten LW, et al. (2006) Effect of oral vitamin B-12 with or without folic acid on cognitive function in older people with mild vitamin B-12 deficiency: a randomized, placebo-controlled trial. Am J Clin Nutr 84, $361-370$.

72. McMahon JA, Green TJ, Skeaff CM, et al. (2006) A controlled trial of homocysteine lowering and cognitive performance. N Engl J Med 354, 2764-2772.

73. Pathansali R, Mangoni AA, Creagh-Brown B, et al. (2006) Effects of folic acid supplementation on psychomotor performance and hemorheology in healthy elderly subjects. Arch Gerontol Geriatr 43, 127-137.

74. Seal EC, Metz J, Flicker L, et al. (2002) A randomized, double-blind, placebo-controlled study of oral vitamin $\mathrm{B}_{12}$ supplementation in older patients with subnormal or borderline serum vitamin $\mathrm{B}_{12}$ concentrations. $J$ Am Geriatr Soc 50, 146-151.

75. van Uffelen JGZ, Chinapaw MJM, van Mechelen W, et al (2008) Walking or vitamin B for cognition in older adults with mild cognitive impairment? A randomised controlled trial. Br J Sports Med 42, 344-351.

76. Aisen PS, Schneider LS, Sano M, et al. (2008) High-dose B vitamin supplementation and cognitive decline in Alzheimer disease: a randomized controlled trial. JAMA 300, 1774-1783.

77. Durga J, van Boxtel MPJ, Schouten EG, et al. (2007) Effect of 3-year folic acid supplementation on cognitive function in older adults in the FACIT trial: a randomised, double blind, controlled trial. Lancet 369, 208-216.

78. Lewerin C, Matousek M, Steen G, et al. (2005) Significant correlations of plasma homocysteine and serum methylmalonic acid with movement and cognitive performance in elderly subjects but no improvement from short-term vitamin therapy: a placebo-controlled randomized study. Am J Clin Nutr 81, 1155-1162.

79. Bryan J, Calvaresi E \& Hughes D (2002) Short-term folate, vitamin B-12 or vitamin B-6 supplementation slightly affects memory performance but not mood in women of various ages. J Nutr 132, 1345-1356.

80. Maylor EA, Simpson EEA, Secker DL, et al. (2006) Effects of zinc supplementation on cognitive function in healthy middle-aged and older adults: the ZENITH study. Br J Nutr 96, $752-760$.

81. Murray-Kolb LE \& Beard JL (2007) Iron treatment normalizes cognitive functioning in young women. Am J Clin Nutr 85, 778-787.

82. Kessler H, Pajonk FG, Bach D, et al. (2008) Effect of copper intake on CSF parameters in patients with mild Alzheimer's disease: a pilot phase 2 clinical trial. J Neural Transm 115, $1651-1659$.

83. Smith A, Clark R, Nutt D, et al. (1999) Anti-oxidant vitamins and mental performance of the elderly. Hum Psychopharmacol Clin Exp 14, 459-471.

84. Casini ML, Marelli G, Papaleo E, et al. (2006) Psychological assessment of the effects of treatment with 
phytoestrogens on postmenopausal women: a randomized, double-blind, crossover, placebo-controlled study. Fertil Steril 85, 972-978.

85. Howes JB, Bray K, Lorenz L, et al. (2004) The effects of dietary supplementation with isoflavones from red clover on cognitive function in postmenopausal women. Climacteric 7, 70-77.

86. Kritz-Silverstein D, Von Muhlen D, Barrett-Connor E, et al. (2003) Isoflavones and cognitive function in older women: the SOy and Postmenopausal Health In Aging (SOPHIA) Study. Menopause 10, 196-202.

87. Francis ST, Head K, Morris PG, et al. (2006) The effect of flavanol-rich cocoa on the fMRI response to a cognitive task in healthy young people. J Cardiovasc Pharmacol 47, S215-S220.

88. Mix JA \& Crews WD (2000) An examination of the efficacy of Ginkgo biloba extract EGb 761 on the neuropsychologic functioning of cognitively intact older adults. J Altern Complement Med 6, 219-229.

89. Woo J, Lau E, Ho SC, et al. (2003) Comparison of Pueraria lobata with hormone replacement therapy in treating the adverse health consequences of menopause. Menopause 10, 352-361.

90. Ryan J, Croft K, Mori T, et al. (2008) An examination of the effects of the antioxidant Pycnogenol ${ }^{\circledR}$ on cognitive performance, serum lipid profile, endocrinological and oxidative stress biomarkers in an elderly population. J Psychopharmacol 22, 553-562.

91. Santos RF, Galduróz JC, Barbieri A, et al. (2003) Cognitive performance, SPECT, and blood viscosity in elderly nondemented people using Ginkgo biloba. Pharmacopsychiatry 36, 127-133.

92. Stough C, Clarke J, Lloyd J, et al. (2001) Neuropsychological changes after 30-day Ginkgo biloba administration in healthy participants. Int J Neuropsychopharmacol 4, $131-134$.

93. Wesnes KA, Simpson P \& Christmas L (1989) A microcomputerised system for evaluating the cognitive actions of drugs in the young, elderly and demented. Eur $J$ Clin Pharmacol 36, A38.

94. Detterman DK (1990) Computerized cognitive abilities tests for research and teaching. Micro Psychol 4, 51-62.

95. Reitan RM (1992) Trail Making Test Manual for Administration and Scoring. Tucson, AZ: Reitan Neuropsychology Laboratory.

96. Wechsler D (1987) Manual for the Wechsler Memory ScaleRevised. San Antonio: The Psychological Corporation.

97. Wechsler D (1981) WAIS-R: Manual: Wechsler Adult Intelligence Scale-Revised. New York: The Psychological Corporation.

98. Hoyland A, Lawton CL \& Dye L (2008) Acute effects of macronutrient manipulations on cognitive test performance in healthy young adults: a systematic research review. Neurosci Biobehav Rev 32, 72-85.
99. Williams CM, El Mohsen MA, Vauzour D, et al. (2008) Blueberry-induced changes in spatial working memory correlate with changes in hippocampal CREB phosphorylation and brain-derived neurotrophic factor (BDNF) levels. Free Radic Biol Med 45, 295-305.

100. Shukitt-Hale B, Cheng V \& Joseph JA (2009) Effects of blackberries on motor and cognitive function in aged rats. Nutr Neurosci 12, 135-140.

101. Devan BD, Goad EH \& Petri HL (1996) Dissociation of hippocampal and striatal contributions to spatial navigation in the water maze. Neurobiol Learning Mem 66, 305.

102. McDonald RJ \& White NM (1994) Parallel information processing in the water maze: evidence for independent memory systems involving dorsal striatum and hippocampus. Behav Neural Biol 61, 260-270.

103. Zyzak DR, Otto T, Eichenbaum H, et al. (1995) Cognitive decline associated with normal aging in rats: a neuropsychological approach. Learn Mem 2, 1-16.

104. Andres-Lacueva C, Shukitt-Hale B, Galli RL, et al. (2005) Anthocyanins in aged blueberry-fed rats are found centrally and may enhance memory. Nutr Neurosci 8, 111-120.

105. Youdim KA, Shukitt-Hale B \& Joseph JA (2004) Flavonoids and the brain: interactions at the blood-brain barrier and their physiological effects on the central nervous system. Free Radic Biol Med 37, 1683-1693.

106. Astur RS, Taylor LB, Mamelak AN, et al. (2002) Humans with hippocampus damage display severe spatial memory impairments in a virtual Morris water task. Behav Brain Res 132, 77-84.

107. Schacter DL (1987) Implicit memory: history and current status. J Exp Psychol Learn Mem Cogn 13, 501-518.

108. Anderson JR (1983) The Architecture of Cognition. Cambridge, MA: Harvard University Press.

109. Poldrack RA \& Packard MG (2003) Competition among multiple memory systems: converging evidence from animal and human brain studies. Neuropsychologia 41, 245-251.

110. Buckner RL, Petersen SE, Ojemann JG, et al. (1995) Functional anatomical studies of explicit and implicit memory retrieval tasks. J Neurosci 15, 12-29.

111. McDaniel MA, Einstein GO \& Rendell PG (2008) The puzzle of inconsistent declines in prospective memory: a multiprocess explanation. In Prospective Memory: Cognitive, Neuroscience, Developmental and Applied Perspectives, pp. 141-160 [M Kliegel, MA McDaniel and GO Einstein, editors]. New York, USA: Lawrence Erlbaum Associates.

112. Burgess PW, Quayle A \& Frith CD (2001) Brain regions involved in prospective memory as determined by positron emission tomography. Neuropsychologia 39, 545-555.

113. Meikle A, Riby LM \& Stollery B (2004) The impact of glucose ingestion and gluco-regulatory control on cognitive performance: a comparison of younger and middle aged adults. Hum Psychopharmacol 19, 523-535. 